\title{
Appearance Modeling of Living Human Tissues
}

\author{
Augusto L.P. Nunes ${ }^{1,2}$, Anderson Maciel $^{2}$, Gary W. Meyer ${ }^{3}$, Nigel W. John ${ }^{4}$, Gladimir V.G. Baranoski ${ }^{5}$, Marcelo Walter ${ }^{2}$ \\ ${ }^{1}$ Federal Institute of Paraná, Londrina, Brazil \\ ${ }^{2}$ Institute of Informatics, Universidade Federal do Rio Grande do Sul, Brazil \\ ${ }^{3}$ Dept. of Computer Science and Engineering, University of Minnesota, USA \\ ${ }^{4}$ Dept. of Computer Science, University of Chester, UK \\ ${ }^{5}$ Natural Phenomena Simulation Group, D.R. Cheriton School of Computer Science, University of Waterloo, Canada
}

\begin{abstract}
The visual fidelity of realistic renderings in Computer Graphics depends fundamentally upon how we model the appearance of objects resulting from the interaction between light and matter reaching the eye. In this paper, we survey the research addressing appearance modeling of living human tissue. Among the many classes of natural materials already researched in Computer Graphics, living human tissues such as blood and skin have recently seen an increase in attention from graphics research. There is already an incipient but substantial body of literature on this topic, but we also lack a structured review as presented here. We introduce a classification for the approaches using the four types of human tissues as classifiers. We show a growing trend of solutions that use first principles from Physics and Biology as fundamental knowledge upon which the models are built. The organic quality of visual results provided by these Biophysical approaches is mainly determined by the optical properties of biophysical components interacting with light. Beyond just picture making, these models can be used in predictive simulations, with the potential for impact in many other areas.

CCS Concepts

-Computing methodologies $\rightarrow$ Reflectance modeling; Texturing;
\end{abstract}

pdfauthor=Augusto L.P. Nunes and colleagues, pdftitle=Appearance Modeling of Living Human Tissues

\section{Introduction}

Together with light and a viewer, objects and their material properties comprise the triad of elements needed to render a virtual scene. These properties explain how light interacts with the subject matter and allow for increased levels of visual realism. The digital modeling of appearance [DRS08] has evolved into a sophisticated specialization inside computer graphics, responsible for many of the advances in photographic quality of renderings [GXZ ${ }^{*} 13, \mathrm{KSZ}^{*} 15$, LDHM16, $\left.E^{*} 05\right]$. Throughout this paper we assume the definition of object appearance from Hunter and Harold [HH87], which involves materials spectral propagated light and its spatial energy distribution.

The natural world has intrinsic properties that complicate its modeling for computer graphics purposes. Living human tissue is one part of the natural world where the complications scale up, considering their dynamic nature and low accessibility. Thus, specialized solutions are often required. In this work, our goal is to present a survey of research targeting living digital human tissue representations, particularly for rendering purposes.
Material modeling of living human tissue has many applications, from increasing realism in the entertainment industry and photorealistic digital humans to medical applications. Medical applications have a long tradition of benefiting from computer graphics research [RRG $\left.{ }^{*} 76\right]$ [SG78]. This is an increasingly important aspect of the new tools becoming available for anatomy education, medical procedures training, and surgical rehearsal and planning. These applications typically make use of medical scan data, which provide $2 \mathrm{D}$ greyscale images.

Techniques such as surface and volume rendering allow us to create 3D representations of the scan data but the question remains on how to provide accurate color and material properties. There are some color data from sources such as the Visible Human project [Ack98], which provides cross-sectional photographs of the human body. Such data is useful but does not solve the material appearance representation problem for all living human tissues, especially when we take into account the medical area demands.

For surgical training, and indeed training of all clinical specialties, the gold standard for teaching human anatomy for hundreds of years has been the dissection of cadaveric specimens, despite the appearance and other properties of cadaveric tissue being quite different from when the tissue cells were still living. This remains an 
important tool, but medical schools are more and more using multiple pedagogical resources that complement each other, including the use of prosections, plastination, and digital tools. Estai [EB16] provides a critical review of current teaching practices in anatomy education and recognizes that full body dissections are still needed for those with surgical career intentions but with a need to integrate new methodologies into the curriculum alongside. One emerging digital tool are virtual anatomy tables such as the Anatomage Table (Anatomage Inc., San Jose, CA) and Sectra Terminals (Sectra AB, Linkoping, Sweden) - see Figure 1. These use medical image scans and histology data to provide a 3D rendering of human anatomy at a real-life scale. The anatomy structure will be accurate, but the colors used for the renderings have to be approximated as the source images are typically grey scale. Many of the techniques reported in this paper could therefore be applied, but this is not generally the case and a user-defined false color map is applied to the renderings.

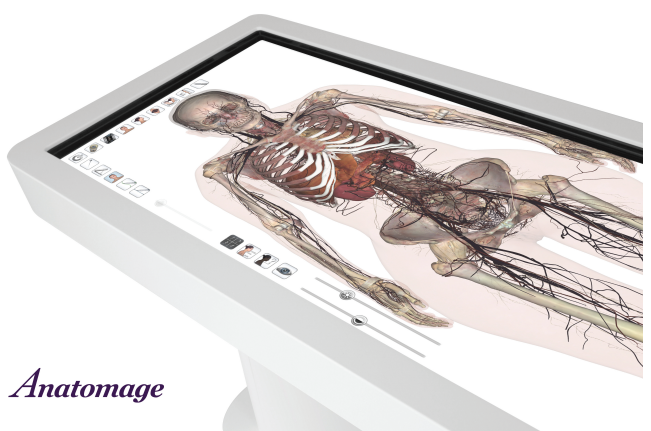

Figure 1: A Virtual Dissection Table. Image courtesy of Anatomage Inc.

Another trend is the increasing number of surgeries performed using Minimally Invasive Surgery (MIS) techniques, and the accepted opinion is that surgeons should attempt to perform the least invasive surgery possible [HVFFTPS12]. On one hand, MIS can significantly reduce the overall operational cost of performing surgeries [TSM $\left.{ }^{*} 15\right]$, but are more complex than traditional open surgery, demanding many years of training in new skills [EL13]. Virtual simulators are therefore necessary tools to scale the access to MIS training to the large demand for experienced professionals. These simulators combine a number of desired properties beyond just visualization, such as interaction, shape modeling, and physics-based dynamic behavior. There is evidence that, as the fidelity of simulation increases, so does the acquisition of skills by professionals [AMD*10]. Minimally invasive surgery is carried out based on a video stream obtained from an endoscopic camera inserted into the patient. To model a MIS scenario, which includes robotic surgery, requires the computer-based renderings to closely resemble what would be seen on this video footage. Again, many techniques here presented are ideal for this need.

In the remainder, we first present biological background on human tissues and their types (Section 2). This background helps contextualize the many approaches and also strengths the crossdisciplinary nature of this review. In Section 3, we illustrate the light-material interaction considering typical human tissues and we propose modeling classes to characterize the reviewed methods according to their motivation, i.e., their mathematical approximation assumptions. From Section 4 through 9, we detail all approaches classified according to the biological definition of human tissues, for living tissue only. Section 10 presents a summary of interesting applications, conclusions, and possible directions for future research.

Modeling the appearance of the human body, both internal and external, is without question an old and permanent challenge for graphics research. We believe this survey will ease this task by providing a taxonomy and review of current approaches, which is a task that has not been carried out before. This will hopefully encourage fresh results from both old and new practitioners in this exciting area.

\section{Biological Background}

Considering the constituent elements of the human body, at the lowest unit level we have the cells, around 100 trillion of them [GH06]. At the highest organizational level, we have the many systems - respiratory, digestive, etc. - which are, in turn, composed of organs that together are responsible for particular functions.

Tissues are at an intermediate scale, being composed of many cells similar in structure and function, typically of the same type, but not necessarily. Tissues are organized into organs which comprise the human body. A single blood vessel, for instance, is made of three types of tissues as shown in Figure 2.

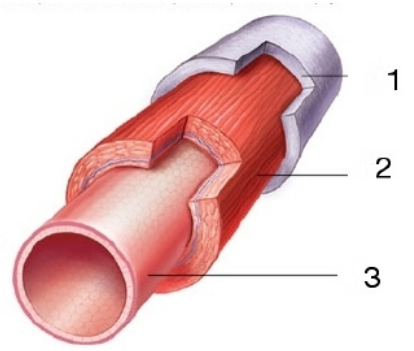

Figure 2: Organs are made up of different tissues. Blood vessel: (1) Connective (2) Muscle (3) Ephitelial. Source: www.pathwayz.org

The set of chemical components that form the human body includes both organic and inorganic materials. The interaction between these originates new composite materials, such as tissues. Some tissues are dead, like hair and nails. They are simply a cell agglomerate without any metabolic or biochemical functions and appear outside the human body. They can be easily removed and once removed they do not change their visual appearance. Therefore they are relatively easier to represent and amenable to computer simulations, as the many advances in the rendering of hair show [KM17]. On the other hand, living tissue has intrinsic dynamic characteristics that make it harder to address in graphics research. Besides, most tissues are located inside the human body and are therefore difficult to access.

There are four basic types of human tissues [Mar15], as depicted in Figure 3, which have specific functions and appearance: 
- Epithelial: the main function of epithelial tissue is the lining of surface or body cavities, and also glandular secretion. They are formed by aggregated polyhedral cells that can be flat, cuboidal, or columnar.

- Connective: the most abundant type in the body. Its main function is support and protection of organs. Bones and cartilages are made up of connective tissue. Blood is also a connective tissue, although quite peculiar compared to other connective tissues. It is also the only fluid tissue in the body.

- Muscle: its main function is to enable body movements. There are three types: (i) skeletal, which attaches to bones and constitutes the muscular system; (ii) smooth muscle tissue, found in the walls of hollow organs and responsible for transporting material through organs; (iii) heart or cardiac tissue, lining the heart wall.

- Nervous: its main function is the transmission of brain impulses with neural cells called neurons. Brain, spinal cord, and nerves are all made up of nervous tissue.

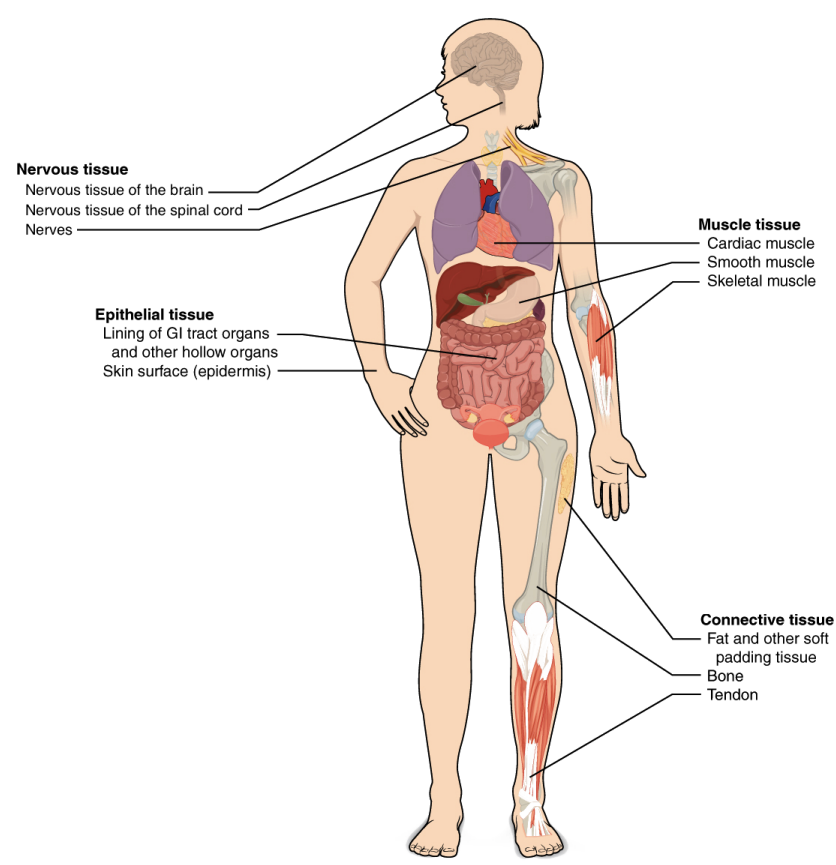

Figure 3: Types and examples of the four tissues present in the human body. Source: www.khanacademy.org

\section{Modeling Approaches for Light-Matter Interaction}

When light interacts with any matter, it can be reflected, transmitted, scattered, and absorbed. In many cases a layered structure is used to model the material and the Kubelka-Munk theory has been used in Computer Graphics to model these interactions [HM92] [DH96]. Typical human tissues have also a layered structure of inhomogeneous media composed of specific biophysical elements whose geometry, spatial distribution, and spectral responses drive the tissue appearance [JWJCvG11]

A typical light-material interaction for a tissue under standard lighting conditions has incident rays $\omega_{i}$ over a surface point $x_{i}$ with normal $N$. This first local interaction can produce a light reflection for some outgoing direction $\omega_{o}$. For layered materials the light may follow different optical paths. In this case, if a biophysical element (with its optical properties) interacts with the light beam we have two possibilities: scattering and absorption. Scattering events can eventually redirect the ray for an arbitrary output point $x_{o}$ over the surface, otherwise, an absorption event can stop the light ray interactions [BK10]. In this context, the final surface color is a result of a biological process.

The above described process can be physically described by a Bidirectional Subsurface Scattering Distribution Function (BSSRDF) [NRH $\left.{ }^{*} 92\right]$. It has both directional and positional dependencies for a light beam integrated over an incoming solid angle $d \omega_{i}$, as depicted in Figure 4.

A simpler version of BSSRDF called Bidirectional Reflectance Distribution Function (BRDF) is regarded as a BSSRDF without positional dependence, that is, $x_{i}=x_{o}$. If we add a spatial dependency we obtain a Spatially Varying Bidirectional Reflectance Distribution Function (SVBRDF). Throughout the paper, when appropriate, we point out models whose formulation enables the effective characterization of light-material interaction in terms of the above quantities. (a)

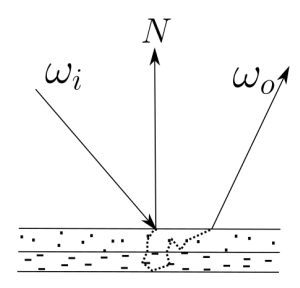

(b)

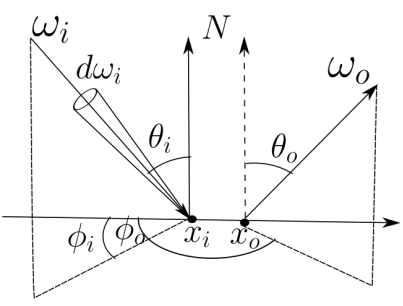

Figure 4: Light interaction with a typical tissue structure (a) and the BSSRDF geometry (b).

To meet our survey goals, we classify the literature on appearance modeling not only according to the tissue type they represent, but also according to the Computer Graphics approach used do model them. Therefore, inspired by the literature about material appearance modeling [Fer03, DRS08, BK10], we define four classes as follows:

- Functional Models: This class provides solutions that convey visual information necessary to complete a task, like a laparoscopy surgery simulator, but do not target high visual realism. Dorsey and colleagues call them empirical models in their book [DRS08] . We extend Ferwerda's original definition [Fer03] to characterize approaches that use simple and generic lighting models, such as Lambertian Reflection, Phong's specular term and so on.

- Biophysically Inspired Models: These models were designed to be primarily employed in the generation of believable images without attempting to accurately simulate the specific biophysical processes responsible for tissue appearance attributes. Con- 
sequently, their evaluations did not go beyond the visual inspection of the resulting images [BK10]; they aim at photorealism.

- Physically Based Models: Considering [Fer03], the formulation of the models in this class describes physical processes for light-matter interaction to represent appearance. Therefore, they implement physics constraints such as energy conservation. Besides, they can directly apply actual data or fit lighting models parameters. The approaches in this category use purely geometric information to derive their results.

- Biophysically Based Models: We include, in this class, models which accurately simulate fundamental photobiophysical processes that determine the appearance of tissues. Besides, these models apply important tissue optics concepts for reproducing spatial and spectral responses comparable to actual data in a parametric formulation with regards to the tissue biophysical compounds optical properties [BK10]. Besides geometric information, the models in this class also use dynamic biophysical processes to model light-matter interaction.

From Functional to Biophysically Based models, we observe increasing complexity in the modeling process. Later, in Section 10, we discuss how these classes evolved over time, and what we can expect from future research in this area.

\section{Human Tissues and their Modeling}

In Table 1, we present a summary of the surveyed approaches, organized by tissue types and modeling classes. In this summary, we only included research where the organs tissue appearance was addressed. For some tissues, particularly muscles, there is an extensive body of research that addresses their physical behavior, mostly for computer animation purposes [ISYM15, KPL*16]. Since our focus is on the appearance modeling, these approaches were not included. Furthermore, optical properties of human organic materials vary individually due to factors such as disorders, habits, and emotions. Therefore, we included works about these dynamic appearance effects in our review. We treat death as a limit for our search. Thus, tissues such as hair [KK89, $\left.\mathrm{MJC}^{*} 03, \mathrm{WBK}^{*} 07\right]$, fur [YTJR15], and nails, which are skin appendages of dead cells, were not included.

For computer graphics goals and digital material representation, we will argue that we should target the mesoscale present in tissues. Maybe an analogy will help. If we think of rendering a house, computer graphics has approaches for modeling of appearance based on the many materials needed to make the house. We know how to render wood, metals, glass, and so on. We think of appearance modeling in terms of the many materials needed to render the objects as needed. We do not focus on the objects themselves, such as doors or furniture; we focus on the materials needed to make these. We think we need a similar strategy for human living tissue. It is important to note, however, that we do not think that a tissue-based approach will guarantee the same flexibility we now have for nonorganic material modeling. We do not expect, for instance, that solutions for one organ of a particular type of tissue will automatically translate into a solution for another organ. Nevertheless, we can expect methodologies and models to be similar, benefiting the area as a whole.

Another advantage of a tissue-based approach is that models can be incrementally built. Let us consider the previously mentioned blood vessel, composed of 3 different tissues. An initial model could consider only the outer connective layer as a constituent element. The other two layers made of muscle and epithelial tissue could be added as required, considering the visual quality of results as a metric.

Our taxonomy for the reviewed papers, i.e., the classes of tissue types, were populated according to the organ tissue which was actually modeled. Since organs often present multiples types of tissues in their composition, we classified the reviewed methods taking into account the organ structure to identify the main tissue that was simulated.

Below, we detail the many approaches summarized in Table 1. In the next sections, we organized the different methods for modeling of light-material interactions by tissue type. We then concisely examine research representative of each methodology underscoring the organ descriptions employed by them. For consistency purposes, we initially provide a brief overview of each organ main morphological characteristics.

\section{Epithelial Tissue}

The epithelium is a continuous sheet of cells over a basement membrane which protects cavities and organs from infections [ONN13]. This tissue has different shapes of component cells organized from simple to stratified layers, where the skin is an instance which has been the object of different lines of research in computer graphics, from models to compute its appearance attributes (e.g., [INN07, BK10]) and techniques to capture these attributes from living subjects (e.g., [MWL $\left.{ }^{*} 99, \mathrm{WMP}^{*} 06, \mathrm{GHP}^{*} 08, \mathrm{JSB}^{*} 10\right]$ ) to algorithms to simulate variations on its appearance over time (e.g., [BW17, Che15, IGAJG15]), just to name a few. In fact, a comprehensive review of the extensive body of work aiming at the rendering of skin would merit a state-of-the-art report in its own right, contributing to recent efforts as in [KRP* 15]. Clearly, such a review would be beyond the scope of this paper centered on how the distinct representations of human tissues affect the modeling of their appearance. Accordingly, in this section, we loosely organize the different methodologies used in the modeling of light and skin interactions. In addition, we report the methods for representing epithelial tissue of internal organs such as bronchus and uterus.

\subsection{Skin}

Human skin is usually described as being composed of three distinct tissues, namely stratum corneum, epidermis and dermis [YTY09]. The stratum corneum and the epidermal layers (stratum lucidum, stratum granulosum, stratum spinosum and stratum basale, from the outermost to the innermost) are predominantly composed of stratified cells [AP82]. It is worth noting that the stratum lucidum is a clear layer found only in thick skin regions such as palms and soles [dG95].

Melanin, arguably the main light absorber found in human skin, is synthesized by melanocyte cells in the stratum basale, where it is preferentially concentrated [dG95]. As the epidermal cells move upward, it is distributed throughout the full thickness of the upper 


\begin{tabular}{|c|c|c|c|c|c|}
\hline \multirow{2}{*}{ Tissue } & \multirow{2}{*}{ Organ } & \multicolumn{4}{|c|}{ Modeling } \\
\hline & & Functional & Biophysically Inspired & Physically Based & Biophysically Based \\
\hline \multirow{3}{*}{$\begin{array}{l}\text { Epithelium } \\
\text { (section 5) }\end{array}$} & Skin & - & $\begin{array}{c}\text { [HK93] [Sta01] } \\
{[\text { JMLH01] [JB02] }} \\
{[\mathrm{DJ} 05][\mathrm{INN07]}} \\
{[\mathrm{dLE} 07]\left[\mathrm{DWd}^{*} 08\right]} \\
{\left[\mathrm{JSB}^{*} 10\right][\mathrm{BW} 17]} \\
\end{array}$ & $\begin{array}{c}{\left[\mathrm{MWL}^{*} 99\right]} \\
{\left[\mathrm{WMP}^{*} 06\right]} \\
{\left[\mathrm{GHP}^{*} 08\right]} \\
{\left[\mathrm{GTB}^{*} 13\right][\mathrm{AS} 17]}\end{array}$ & $\begin{array}{c}\text { [KB04] [DJ06] } \\
\text { [CBKM15] [Che15] } \\
{[\text { IGAJG15] [BVC17] }}\end{array}$ \\
\hline & Bronchus & - & - & $\begin{array}{l}{\left[\mathrm{CDS}^{*} 04\right]} \\
{\left[\mathrm{CDS}^{*} 06\right]} \\
\end{array}$ & - \\
\hline & Uterus & - & {$\left[\mathrm{BZH}^{*} 05\right]$} & [MB12] [MB14] & - \\
\hline \multirow{4}{*}{ Connective (section 6) } & Teeth & - & $\begin{array}{c}\text { [Gon10] [SB10] } \\
\text { [LFJB12] [JMDH12] } \\
\text { [Jun16] }\end{array}$ & - & - \\
\hline & Blood & $\begin{array}{c}\text { [BCHSS99] } \\
\text { [HSD10] } \\
{[\text { BMPG11] }} \\
\text { [BPBG13] [UF15] } \\
\text { [AMS*15] } \\
\end{array}$ & - & - & {$\left[\mathrm{YBK}^{*} 12\right]$} \\
\hline & $\begin{array}{c}\text { Bones, } \\
\text { Ligaments, } \\
\text { Cartilage } \\
\end{array}$ & $\begin{array}{l}\text { [sCHRU85] } \\
{[\mathrm{ECBH06]}} \\
{[\mathrm{DGBW09]}} \\
\end{array}$ & [KSS09] [DHF*16] & - & - \\
\hline & Liver & [KMKK88] & $\begin{array}{c}\mathrm{MCT}^{*} \text { 98] [NC99] } \\
{\left[\mathrm{NHS}^{2}\right]\left[\mathrm{HLX}^{*} \text { 09] }\right.} \\
{[\mathrm{LWHL} 12]}\end{array}$ & [NMCW17] & - \\
\hline Muscle (section 7) & Eye & [SBMH94] & $\begin{array}{l}{\left[\mathrm{LBS}^{*} 03\right][\mathrm{FGBB} 09]} \\
{\left[\mathrm{BBN}^{*} 14\right][\mathrm{BBGB} 16]}\end{array}$ & - & [WLVB06] [KBC* 17a] \\
\hline Nervous (section 8) & Brain & - & - & $\begin{array}{l}\text { [aCWJ*11] } \\
{\left[\mathrm{aCJB}^{*} 12\right]} \\
\end{array}$ & - \\
\hline \multicolumn{2}{|c|}{ General Approaches (section 9) } & $\begin{array}{c}\text { [SG78] [Rob99] } \\
{\left[\mathrm{SBD}^{*} 00\right]}\end{array}$ & $\begin{array}{l}\text { [ELDzY04] [E*05] } \\
\text { [LJD07] [BTM17] }\end{array}$ & $\begin{array}{l}{\left[\mathrm{QBY}^{*} 15\right]} \\
{\left[\mathrm{QBY}{ }^{*} 16\right]}\end{array}$ & - \\
\hline
\end{tabular}

Table 1: Summary of Approaches for Living Human Tissue Research in Computer Graphics.

epidermal layers [KSZC91]. This pigment may occur in a colloidal form or clustered within organelles called melanosomes [Pat95].

In lightly pigmented specimens, the melanosomes can form clusters surrounded by a transparent membrane known as melanosome complexes, while in darkly pigmented specimens they occur as denser and individually dispersed particles [SGPF69, OGE73]. The presence of melanosomes and melanosome complexes in the cutaneous tissues may result in detour and sieve effects that can affect the probability of light absorption [But64, Lat84].

The dermis forms the bulk of the full thickness of skin. It can be divided into the papillary dermis and reticular dermis [AP82]. Compared to the reticular dermis, the papillary dermis is relatively thin and contains smaller-sized structural collagen fibrils. The dermis also contains a network of blood vessels, with wider vessels being located in the reticular dermis, and its optical properties are largely affected by the presence of hemoglobin and other bloodborne pigments such as bilirubin and beta-carotene.

Eventually, the light traversing the cutaneous tissues may reach the hypodermis. This adipose tissue consists mostly of fat cells that contain a number of smooth droplets of lipids whose size is larger than that of typical tissue scatterers [BGKT05]. Light reflection by these large cellular structures can contribute to the overall scattering of light within the dermis [YTY09].

In the next sections, we introduce a review of skin sampling methods to capture skin appearance, then we approach models to represent skin light-material interaction. Incidentally, the terms "deterministic" and "stochastic" employed in the taxonomy presented in Section 5.1.3 and 5.1.4 refer to whether or not the models possess some inherent randomness [RAG13].

\subsubsection{Appearance Capturing Methods}

Marschner et al. [MWL ${ }^{*} 99$ ] introduced the sampling of skin attributes from living subjects for computer graphics purposes. In their work, the BRDF of skin was measured based on a set of photographs taken in a controlled environment, i.e., with specific lighting and camera poses, besides known geometries. By computing the pictures inverse rendering, the authors could quickly estimate the skin reflectance of a human face. Since then, capturing the skin reflectance has been addressed in many computer graphics works, varying from their acquisition setup to the skin layered structure modeling [DHT*00, WMP*06, GHP*08, AS17].

Jimenez et al. [JSB* 10] presented a method to render facial skin considering the dynamic appearance of emotions, such that, mechanical deformations and involuntary dilatation/constriction of blood vessels impact on the final skin rendering. They acquire hemoglobin distribution over facial expressions of living subjects through the SIAscope ${ }^{\mathrm{TM}}$ system [CCH99], which is an imaging device for skin subsurface sampling under colored and near-infrared polarized lighting. In this context, they computed the histogram of hemoglobin 
distribution maps from every expression and applied a local histogram matching over the original rendering.

Graham et al. [GTB*13] discussed the skin surface geometry description as an important factor for improving the visual realism of renderings. They proposed a technique for skin microstructure synthesis based on scanned patches from high-resolution images of a subject. In small scale, this method computes the surface normal and the BRDF for every pixel in a skin pacth. In fact, each face region was sampled in a different patch which was the input for an extended Ashikhmin algorithm [Ash01] (an example-based texture synthesis technique) to compute the rendering for a new face.

\subsubsection{Biophysically Inspired Models}

The first representative model in this group is the multiple-layer scattering model, henceforth referred to as HK model, proposed by Hanrahan and Krueger [HK93]. This model employs a stochastic formulation based on the application of Monte Carlo methods for simulating light propagation in human tissue [Pra88]. Within its formulation, the skin tissues are represented using two layers, one for the epidermis and the other for the dermis. This model was designed to simulate the scattering behavior of layered organic (e.g., skin and leaves) and inorganic (e.g., snow and sand) materials appearing in nature. Because of its generality, it overlooks specific characteristics and properties of the skin tissues such as the mechanisms of light absorption by pigments like melanin and hemoglobin. As a result, the quantities associated with the spectral distribution of light (e.g., reflectance) and responsible for the skin chromatic attributes are not computed directly. Instead, they are implicitly introduced into the model as input data.

Stam [Sta01] proposed the discrete ordinate model, henceforth referred to as DO model. It employs the discrete ordinate approach for radiative transfer [SC84]. It was also developed to simulate the scattering behavior of different layered materials, including skin. Within its original formulation, the skin tissues are represented by a single layer with constant optical properties. Similar to the previous model, spectral quantities related to skin reflectance are introduced into the model as input parameters, i.e., mechanisms of light absorption by natural pigments were only implicitly considered.

Jensen et al. [JMLH01] proposed a model based on the diffusion theory [Ish78] to simulate the scattering behavior of translucent materials (Figure 5). Within its formulation, the entire skin structure is represented as one medium. This model henceforth referred to as the DT model, employs the diffusion approximation for isotropic media known as the dipole method [BK10]. This approximation consists in placing two point sources relative to the surface, one representing the positive real light located below the surface, and the other representing a negative virtual light positioned above the surface. It is then used to compute the radiant exitance at the propagation point separated by a certain distance from the incidence point. Following these approximations, the scattering behavior of a material sample is quantified in terms of the BSSRDF. The performance of this model was later improved by Jensen and Buhler [JB02] through the incorporation of a two-pass hierarchical algorithm. Recently, Frisvad et al. [FHK14] extended the previous method computing a directional ray source diffusion instead of a point source diffusion positioned relative to the surface. Similarly to the standard DT model, they compute rays positioned along to the surface normal, but these rays are defined toward the incidence and emergence points on the media. Finally, this method allows to analytically compute subsurface scattering with a better representation for the light-material interaction.

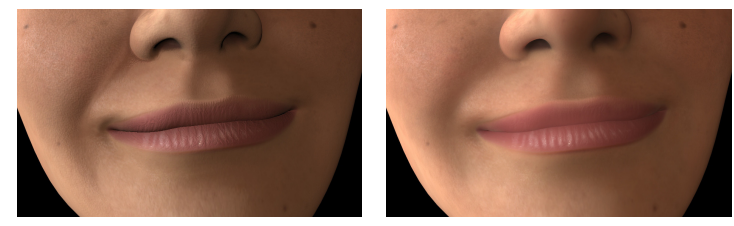

Figure 5: Images of a textured face before (left) and after (right) the addition of the subsurface scattering simulation provided by the DT model [JB02].

Donner and Jensen [DJ05] extended the diffusion approximation used by the DT model [JMLH01] to account for multiple thin layers. For a single thin planar slab within a multi-layered material, diffusion profiles are computed using a multipole (a sum of a number of dipoles). These profiles describe the amount of light reflected and transmitted by the slab considering an infinitesimal focused beam of light impinging on it. Different profiles are computed for each wavelength of interest. To improve performance, d'Eon et al. [dLE07] proposed the approximation of these diffusion profiles by a sum of Gaussians. When applying this framework to the skin, Donner and Jensen [DJ05] consider the skin subdivided into three layers, namely the epidermis, the "upper" dermis and the "bloody" dermis. To characterize each of these layers, they used values for their corresponding volumetric absorption and scattering coefficients available in the tissue optics literature [Tuc00].

Later on, Donner et al. [DWd*08] addressed one of the limitations of previous diffusion theory based models (namely the assumption that skin layers are homogeneous), presenting a layered heterogeneous method for skin appearance representation. More specifically, they employed 2D parameter maps to account for biophysical variations within these tissues. Donner et al. [DWd*08] presented a spectral shading model which expanded upon the work by Donner and Jensen [DJ05] and d'Eon et al. [dLE07]. In their model, skin is represented by two layers, epidermis and dermis, along with an infinitesimally thin absorbing layer between them. In order to characterize the volumetric scattering and absorption coefficients of each layer, they employed formulas provided by Jacques [Jac98]. It is worth noting that these formulas, which were considered tentative approximations by Jacques, overlook relevant light attenuation processes taking place within the skin tissues such as the scattering by the melanosomes in the epidermis and the scattering by the collagen fibrils in the papillary dermis.

\subsubsection{Deterministic Biophysically Based Models}

In a subsequent work, Donner and Jensen [DJ06] applied the multipole method [DJ05] in conjunction with a spectral model to specifically render the appearance of skin. They considered it to be formed by two layers, the epidermis and dermis, and used simple functions to approximate the absorption coefficients of melanin and hemoglobin. These coefficients along with approximation formulas provided by Jacques [Jac98] were used to compute volumetric 
absorption and scattering coefficients for each of the two layers. This model was quantitatively evaluated by comparing modeled reflectance curves with measured curves. However, comparisons involving modeled scattering profiles with measured ones were not performed.

Recently, Iglesias-Guitian et al. [IGAJG15] proposed a spectral model for aging in which skin is represented by five layers, namely stratum corneum, epidermis, papillary dermis, reticular dermis and hypodermis. The volumetric absorption and scattering coefficients for each of these layers are calculated using general formulas provided in the biomedical literature (e.g., [BGT11,Jac13]). These coefficients are then used as input parameters into a general-purpose Monte Carlo algorithm [WJZ95] in order to obtain the diffusion profiles for each of the five layers. Each diffusion profile is transformed from spectral XYZ tri-stimulus values to sRGB color space for rendering. These profiles are given in the form of a sum of six Gaussians (following the method proposed by d'Eon et al. [dLE07]). Finally, these profiles are plugged in the diffusion approximation algorithm proposed by Jensen and Buthler [JB02] to perform the rendering of the skin specimens.

The deterministic formulation employed by models based on the diffusion approximation makes them faster and amenable to analytical manipulation. This is particularly of interest for real-time applications. However, the predictive capabilities of these models are bound by the inherent limitations of the analytical approximations employed in their formulations. For example, it has been demonstrated [HJWT95, CSS01] that the diffusion theory approximation fails to accurately describe light propagation in highly absorbing media. In the case of human skin, strong absorption is verified in the UV, visible (below 600 $\mathrm{nm}$ ), and IR (above 1300 $\mathrm{nm}$ ) domains [AP82]. Although algorithms have been proposed to relax the limitations of the diffusion theory approximation [dI11], it is important to note that these algorithms have been evaluated through theoretical comparisons of their predictions with simulated results obtained using a general-purpose Monte Carlo algorithm [WJZ95] and considering single- and two-layer homogeneous materials. Direct comparisons with skin measured data were not included in the assessment of their biophysical fidelity.

\subsubsection{Stochastic Biophysically Based Models}

Stochastic light transport algorithms allow for the proper modeling of complex geometries and optical inhomogeneities [Pra88, WJZ95], thus supporting the utilization of first principles simulation approaches. These approaches, in turn, apply fundamental principles of physics to a material's microscopic structure in order to build up the bulk behavior of how it interacts with light [DRS08].

Krishnaswamy and Baranoski [KB04] proposed the first model to employ first principles approach for computing both spectral and scattering quantities for skin specimens. This model, known as BioSpec (Biophysically based Spectral model), employs ray optics and Monte Carlo techniques to simulate the processes of light propagation and attenuation in the skin tissues. It considers the stratification of skin into four layers: stratum corneum, epidermis, papillary dermis, and reticular dermis. In addition to the variation of melanin pigmentation, this method is capable of representing skin disorders like reddening (in a site of the skin due to an inflammation).

Recently, Chen et al. [CBKM15] presented the first hyperspectral skin appearance model, henceforth referred to as HyLIoS (Hyperspectral Light Impingement on Skin), capable of predictively simulating both the spectral and spatial distributions of light interacting with this complex biological material in the ultraviolet, visible, and infrared domains, from 250-2500 $\mathrm{nm}$ (Figure 6). Similar to BioSpec, HyLIoS employs stochastic techniques within a first-principles framework. Within this framework, skin is represented by seven layers (stratum corneum, stratum granulosum, stratum spinosum, stratum basale, papillary dermis and reticular dermis). The role played by the hypodermis on the skin hyperspectral responses is also taken into consideration.

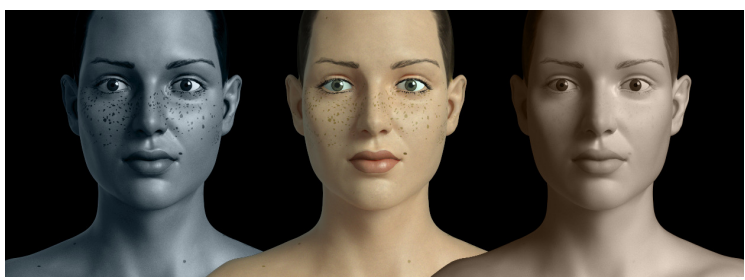

Figure 6: Images generated using the HyLIoS model [CBKM15] to illustrate distinct skin appearance features in the ultraviolet (left), visible (center) and infrared (right) spectral domain. The ultraviolet and infrared spectral responses are depicted in pseudo color.

HyLIoS is the first model to explicitly account for the particle nature of the melanosomes. Their size, shape, orientation, and distribution are incorporated into the simulations using a probabilist strategy [KB07]. Using this strategy, melanosomes (and melanosome complexes) are generated, positioned, and oriented on-the-fly in a given epidermal layer. This approach enables the simulation of light interactions with each melanosome without having to explicitly store these organelles. Consequently, HyLIoS accounts not only for light detour and sieve effects [But64, Lat84], but also the physical distances traveled by light within these tissues. Hence, the radiometric responses derived from the light interaction processes affecting skin appearance can be quantified in terms of BSSRDF since the distances that may separate the light incidence and outgoing points are explicitly provided by the simulations.

It is worth noting that both BioSpec and HyLIoS take into account the presence of collagen fibrils in the papillary dermis and comprehensively simulate the putative occurrence of Rayleigh scattering in this tissue. More specifically, both models compute the corresponding Rayleigh scattering coefficient as a function of the fibril's radius and then adjust the direction of light propagating in this tissue accordingly. These features proved to be essential for the identification of the biophysical mechanisms responsible for the bluish appearance of cyanotic skin [BVC17] and subcutaneous veins [SRVL18].

Depending on the level of abstraction employed in the characterization of the target material, the results provided by stochastic models of light transport can have a high degree of predictability. This aspect was verified in both models outlined above, which 
have been extensively evaluated through comparisons of their predictions with actual measured data. Due to their nature, however, stochastic models can be substantially more time-consuming than their counterparts. Consequently, in order to make them suitable for applications requiring high interactive rates, it is often necessary to employ parallel processing strategies and specialized graphics hardware.

\subsubsection{Dynamic Appearance for Skin}

Skin is the epithelial tissue which received most attention for dynamic appearance representation. As mentioned in the above sections, Computer Graphics methods represent from skin aging [IGAJG15] to facial hemoglobin distribution caused by emotions [JSB $\left.{ }^{*} 10\right]$. Besides, some works approached skin pigmentation disorders in visible [KB04] and non-visible [CBKM15] lighting. For instance, Barros [BW17] presented a method to simulate hyperpigmented and hypopigmented skin lesions using reaction-diffusion for computing local melanosomes concentrations. This model output is suitable with layered models for skin as [JMLH01] or even for texture synthesis. If we know how to control oxygen levels in human blood and understand their relation to human face color, we can apply this knowledge to enhance the appearance of virtual humans [AMS* 15, ABMH16].

Feess and colleagues [FKM16] introduced a wound healing model based on biochemistry and biomechanics that simulates the deformations of skin and formation of scars during the healing process. For skin tissue, they consider the fibrotic appearance of the scars due to the collagen type and represent it by diffusion equations. In another recent work, Choi et al. [CGC17] used biological information about the skin pigmentation to synthesize the appearance of scars. They presented a method to estimate, from reference images computed by inverse-lighting, scar properties such as melanin and hemoglobin concentrations. These rendering parameters together with a tessellation process expresses the scar geometry and appearance over a target image of actual skin. Finally, Chen [Che15] proposed the first physiologically based framework for the simulation and visualization of skin tanning dynamics. It allows for distinct skin appearance models (e.g., BioSpec or HyLIoS) to be employed in the rendering of images depicting tanned subjects.

\subsection{Bronchus}

In the human respiratory system, trachea and bronchus have similarities in their internal wall structure as a specialized epithelial tissue lined by a thin mucous layer. Under this layer, longitudinal folds formed by elastic fibers have nervous and glands permeated by small arteries and lymph vessels. A dense cartilage layer covered by connective tissue composes the bronchus external tunic. The bronchus innermost layer is a columnar pseudostratified epithelium (i.e., a layered structure with several cells) which has cilia lubricated by secretions containing immunoglobulins, and glycoproteins [ONN13].

For computer graphics, this luminal structure attracts some interest due to the availability of image data from its internal wall captured by endoscopes. To formulate models about bronchus lightmaterial interaction require complete descriptions of this phenomenon, especially from in-vivo samples. Unfortunately, to the best of our knowledge, such data remains unavailable in biomedical reports. On the other hand, data-driven approaches could replicate an expected behavior and appearance.

Chung et al. [CDS*04] measured the BRDF of the bronchi using the images and tracked position obtained from an endoscopy camera. They also used data from a previously captured MRI of the specific patient to complete the data necessary to recover intrinsic visual properties from the surface. In a later work [CDS*06], the authors extended the previous method with statistical texture synthesis for disocclusion removal and assessed the resulting realism in an experiment with 12 users. Although they did not compare synthetic images with real ones, they obtained rather positive results from users classifying images between real and computer generated.

\subsection{Uterus}

Present in female mammalians, the uterus is a luminal structure lined by a mucous membrane over three layers of different tissues. Endometrium, the innermost layer, is a thin columnar epithelial tissue which disappears during the menstrual cycle. The intermediate layer, named myometrium, is a thick smooth muscle tissue with blood vessels and connective tissue. Finally, the mesometrium is a connective tissue covering the organ shape [ONN13].

Three-dimensional representation of uterus is an important issue for ultrasound devices in the Medical Imaging area. For Computer Graphics, however, specific methods are not usual. Nevertheless, Bachofen et al. [BZH* 05] presented a method for uterus tissue rendering in a Hysteroscopy simulator (where the organ lumen is inspected during the surgery) (see Fig. 7). They computed multipass rendering, where Bump Mapping reproduces the tissue roughness and the Blinn-Phong model [Bli77] solves the light-material interaction over a textured surface. Besides, textured quads oriented according to the camera viewpoint modeled tissue fibers and bleeding in real time.

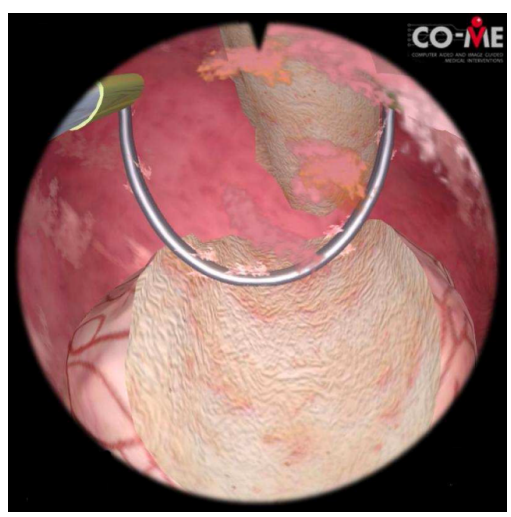

Figure 7: Rendering of the uterus tissue in a Hysteroscopy simulation from $\left[B Z H^{*} 05\right]$.

Malti and Bartoli [MB12] addressed physically plausible lighting models for rendering in vivo organs. They argued that the organs optical properties undergo significant changes when they 
are ex vivo. In this context, they presented a method to estimate the Cook-Torrance [CT82] model parameters using a standard Laparoscopy surgery for sampling organ's BRDF, as the uterus. Although BRDF has four degrees of freedom, they assessed only samples where the light was collinear to the camera direction, assuming that data approximates the material behavior. In 2014, these same authors presented a method to reconstruct deformed human uterus tissue from single view laparoscopy images based on their BRDF measurement technique [MB14]. In fact, their focus was not on rendering, since their concern was mainly the estimation of the organ's shape. Nevertheless, we mentioned these works here because of their specific organic optical properties measurement and their importance for the Cook-Torrance fitting of the uterus tissue material.

\section{Connective tissues}

Connective tissues are a group of tissues responsible for maintaining the form and structure of the body and its organs [Mes17]. The group includes several types of fibrous tissue with different densities and varying cells distributions. Bone, ligaments, tendons, cartilage, and fat are all part of this group, providing cohesion and internal support for all other tissues. The factor that distinguishes the different types of connective tissues and bases their anatomical classification is the relative proportion among its constituents: extracellular fibers (collagen and elastin), stationary and migrating cells, and amorphous ground substance. In each specialized form of connective tissues, one component or another may predominate over all the others, depending upon the local structural or metabolic requirements [OSM16]. This allows for a single prototypic tissue to originate specialized tissues with functions and appearances as diverse as those of blood and fascia - the sheet of fibrous connective tissue that separates the muscles and the organs. One can hypothesize that a common matrix could also be used to model the appearance of these tissues in computer graphics. In this section, we address works where the connective tissue appearance of an organ was the target for their graphics representation method.

\subsection{Human Teeth}

The translucent appearance of a human tooth is determined by the interaction of light with the three different layers of material which compose the tooth. The two outermost layers, enamel and dentin, play the most important role, and all of the attempts to simulate the appearance of teeth have focused on modeling only these two layers. In addition to correctly representing the thickness of the enamel and dentin layers, determining their optical properties is critical for producing a convincing simulation. The external shape of the tooth must also be modeled correctly in order to produce an effective result [Gon10].

For both enamel and dentin, scattering dominates absorption by at least an order of magnitude. The scattering and absorption coefficients of enamel are highly wavelength dependent (blue light is scattered far more than red light), but the same is not true for dentin. There is, however, significant variation in the scattering and absorption properties amongst different members in the human population. The index of refraction is usually taken to be constant across all wavelengths when modeling both enamel and dentin. Scattering from both enamel and dentin is very anisotropic [FGFS95]. Although many attempts to render the appearance of teeth ignore the scattering phase function, other more general efforts employ a modified version of the Henyey-Greenstein function that has fit to measured data.

Shetty and Bailey [SB10] employed the dipole and multipole diffusion approximation technique, developed by Donner and Jensen [DJ05], to simulate the appearance of teeth. While other attempts to recreate the translucent appearance of teeth have selected fixed values for the scattering, absorption, and anisotropy properties, Shetty and Bailey [SB10] left this up to the user's discretion by providing a graphical user interface that allowed people to make adjustments to these parameters. While the range of possible values was determined by data from Fried et al. [FGFS95], the interface had red, green, and blue sliders to set the scattering and absorption for enamel and for dentin. Example renderings produced using this system are shown in Figure 8. One limitation of this work is that it was only possible to make pictures of a single tooth from a limited range of views. This makes it difficult to evaluate circumstances where the diffusion approximation could break down, such as irregular geometry and oblique lighting angles. On the other hand, this appears to be a real-time system.
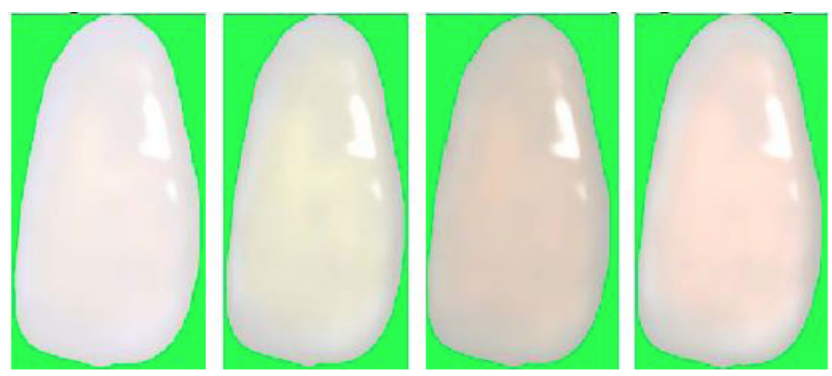

Figure 8: Incisors rendered using Shetty and Bailey [SB10] method with different choices for scattering and absorption properties.

Larsen et al. [LFJB12] also used the multipole technique developed by Donner and Jensen [DJ05], however, they allowed views to be made from a wider range of directions. Just like Shetty and Bailey [SB10], they employed the optical parameters reported by Fried and his colleagues [FGFS95], but they included a coating of saliva on the surface of the teeth as part of their simulation. While their system has greater interactivity because it allows pictures to be made from many different viewpoints the translucent properties of the teeth are not depicted as effectively as in the Shetty and Bailey [SB10] work.

The volume photon mapping method described by Jung et al. [JMDH12] and Jung [Jun16] is the most general approach that has been taken thus far to the rendering of human teeth. Volume photon mapping [JC98] allows subtle light interactions within the tooth to be modeled, but it is computationally expensive to achieve these results and the system does not run in real time. The scattering and absorption spectra given by Spitzer and ten Bosch [StB75], besides data from ten Bosch and Zijp [tBZ87], were employed in this work. Anisotropy and phase function data were taken from Fried $e t$ 
al. [FGFS95], but simplifying assumptions were made in order to get the solution to converge with an acceptable number of photons. A rendering produced using this method that includes the effects of surface and subsurface reflection is shown in Figure 9.

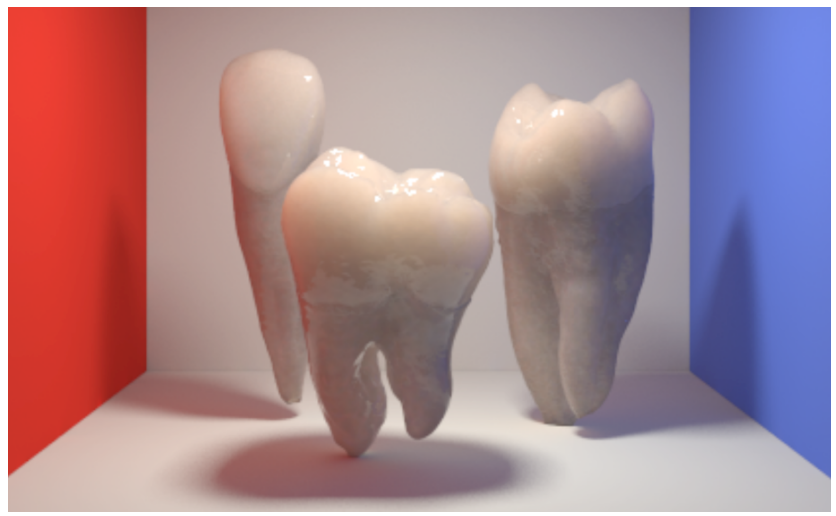

Figure 9: Three different teeth rendered using the volume photon mapping method described in [JMDH12].

\subsection{Blood}

Blood is a very specialized type of connective tissue in which cells are suspended and carried in a fluid, the plasma. Due to its importance in many visual scenarios, the appearance of blood is widely targeted in the literature of computer graphics. However, regardless of the complex interactions occurring between light and blood, the literature focused more on simulating the dynamics of blood as a fluid, often choosing simplistic approaches to model appearance. Basdogan [BCHSS99], for instance, used Navier-Stokes equations to deform a continuous polyhedral surface that is drawn with a red color over the anatomy to simulate bleeding in real time. Besides, blood often played a secondary role in surgery simulations, where any red fluid is enough to convey the presence of blood and enrich the visual of a scalpel cutting through flesh [HSD10,BMPG11, BPBG13,UF15, BTM17]. In realistic rendering, models of light interaction with human skin sometimes account for the presence of blood through the incorporation of absorption coefficients of bloodborne pigments [DJ06, BK10]. Parameters such as oxygenation perfusion and pulse rate have also been used to associate facial skin appearance to the influence of human emotions on blood pigmentation $\left[\mathrm{AMS}^{*} 15\right]$.

As of today, a single work can be found that proposes a predictive model of the whole blood appearance at the level of light interaction with the tissue. The model proposed by Yim et at. [YBK $\left.{ }^{*} 12\right]$ is designed to account for specific morphological, optical and physiological factors affecting light interactions with blood cells. It employs a first principles approach that computes both the spectral (reflectance and transmittance) and spatial (scattering) responses associated to the appearance attributes of blood. Multispectral predictions are obtained and their correctness evaluated in comparison with actual measured data. Modeling in such level allows the technique to be used for both rendering and biomedical applications (see Fig. 10).

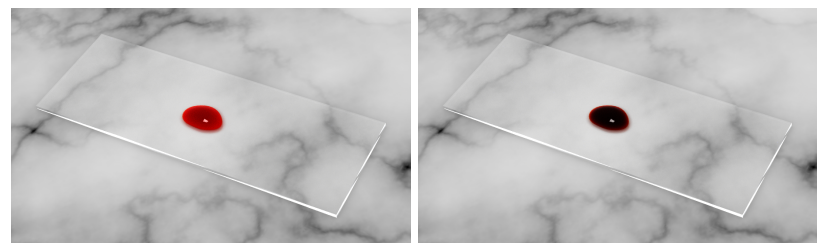

Figure 10: Renderings of blood droplets on a microscope slide showing spectral variations due to different oxygenation levels. Left: oxygenated (arterial) blood. Right: deoxygenated (venous) blood [YBK 12$]$.

\subsection{Bone, Ligaments and Cartilage}

There is a lower demand for photorealistic rendering of bone, ligaments, and cartilage from graphics applications when compared to teeth and blood. This can be explained by the fact that skeletal tissues rarely appear in movies and games, and when they do, there is no need for physically accurate realism. Consequently, very few works approached such tissues in the literature. Besides, while applications in anatomy, biomechanics and pathology use 3D models of skeletal tissues, these structures are often rendered with a non-photorealistic layer to convey density, stress distribution, and thickness with color mapping [SMBT04, MVG04, MM16].

While most graphics applications are based on polygons, bone, cartilage, and ligament are more often found in medical applications, where tomographic data obtained from CT or MRI scanners are rather used. Bone, with anatomical fidelity, first appeared as a rendered object in the middle of the 1980's [sCHRU85]. As the gold standard of the time was the Phong shading method, pioneers struggled to provide methods that offer similar visual quality to Phong's without a polygonal surface.

Today, tomographic 2D axial representations or multiplanar reformations (MPR) are the standard data in daily radiological routine. Nevertheless, medical applications benefit from three-dimensional data in cases where complex anatomy must be conveyed. There, 3D volume rendering [DCH88, Lev88] of CT or MRI data can offer important information that 2D images alone cannot convey for pre-operative planning and post-treatment follow-up [DHF* 16$]$. In such cases, a volume is often used for rendering instead of a polygonal surface model. Classical VRT (volume rendering techniques) use raycasting to sample and integrate intensities from a set of intersecting voxels. The color of an image fragment is then defined by the mapping of these intensities on a transfer function. Surface shading can also be computed using a variety of lighting models and estimates of the surface normals from thresholds in the surface neighborhood [CM09, ZCBM14, CLL*16].

In volume visualization, the data include all body tissues, and a number of parameters must be set to highlight a specific type of tissue. Bone can be easily identified in CT as the image intensities convey material density. Other tissues, including ligament and cartilage, are better represented in MRI. While volume visualization techniques render a remarkably accurate anatomical fidelity, sometimes using global illumination [ZCBM14], none of these techniques focused on modeling connective tissues with physical or biophysical fidelity from the light-matter interaction viewpoint. 
Structural elements have been studied that provide material information for rendering, e.g. distribution of cortical and trabecular bone [DGBW09], and collagen fiber bundles in ligament [ECBH06]. Besides, in surgery simulation, the amount of blood and water on the bone surface has been used to generate wet surfaces as a means to enhance realism [KSS09]. All the same, those works focused on representing tissue structure as a useful visual augmentation for the application rather than visual realism.

Another class of sliced volumetric datasets is that of high-resolution photographic images, such as those from the Visible Human Project dataset [Ack98]. Berndt et al. [BTM17] used position based dynamics to simulate a surgical scenario including bone, soft tissue, and blood. The motion and deformation of solid and fluid materials are solved by a unified approach based on volumetric particles. Then, a surface mesh is computed in real time and colored by sampling the Visible Human Male [SASW96] photographic dataset. The dataset is used as a $3 \mathrm{D}$ texture to provide realistic color for the different connective tissues. Finally, local lighting models are applied for shading in real time.

Back to less invasive imaging, a lot of the information contained in the anatomy tomographic data is ignored by classical volume rendering techniques. More recently, cinematic rendering (CR) techniques [DHF$\left.{ }^{*} 16\right]$ are using neglected information to provide photorealistic rendering to medical image data. Conventional volume rendering considers only emission and absorption of energy along a light ray to calculate $3 \mathrm{D}$ images, modeling scattering effects using a local gradient shading model. CR, instead, solves the multidimensional and non-continuous rendering equation [Kaj86] to integrate the light scattered from all possible directions along a ray. Such path tracing integrates a huge number of light rays to form each pixel of the rendered image. As the number of directions is virtually infinite, Monte-Carlo simulations are used to generate a randomized subset of paths, thus controlling the computations cost. Averaging the numerous Monte-Carlo samples progressively allows obtaining the final image. Moreover, light maps are used for illumination. Using high dynamic range light maps leads to a natural illumination of the rendered data, which contrasts with images generated with conventional volume rendering (Fig. 11). Despite the potential benefits of CR compared to conventional volume rendering, there is a higher computational power demand required for the CR technique because of the more complex lighting model. Rendering of the final image is currently measured in seconds.

The method has a great potential to be quickly integrated in the set of tools used for learning anatomy and also to be used in computer animation tools designed for human anatomy. The potential of $\mathrm{CR}$ for medical applications and any possible advantage in relation to using conventional volume rendering for exams, however, will have to be evaluated by future research [AF16].

\subsection{Liver}

Although the liver is a digestive system organ with restricted access for material sampling (unlike, e.g., skin or eyes), it has attracted a lot of attention in computer graphics research, being the most targeted internal organ. This is probably due to the liver being a parenchymatous organ (i.e, a moderate stiffness volume of functional cells) with a rather stable shape. Besides, the liver has a thin

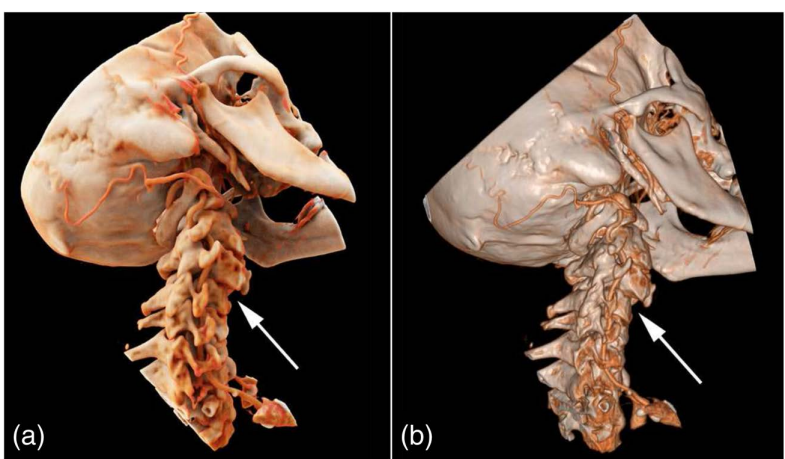

Figure 11: Comparison of the graphical realism obtained with (a) Cinematic Rendering and $(b)$ conventional volume rendering from the same CT data (extracted from [DHF*16]).

connective tissue over its lobes, named Glisson capsule, supporting the organ stroma and parenchyma.

Kashiwagi et al. [KMKK88] introduced the liver 3D visualization as a result from an accurate geometry reconstruction plus a phenomenological lighting model. They computed a single color for every geometric primitive of a triangular mesh reconstructed from CT-Scan images (Flat Shading). For the organic material representation, they assumed it as a Lambertian surface, i.e., considering only the diffuse part of the light-material interaction. Although the results highlight the organ anatomy, the visual aspect of such technique looks like a cartoon, as observed by Satava [Sat95], since the organic tissue has more optical properties than expressed by this model.

Marescaux et al. [MCT*98] addressed the immersion in virtual surgery simulators as a mental effect when the user looks at a 3D image on a screen. To improve the realism of such devices, they reconstructed the liver geometry from the Visible Human Project [Ack98], which has a high-resolution volumetric dataset of the human body. For rendering, this method used Gouraud Shading [Gou71], where colors were assessed for each 3D mesh vertex, computing the Blinn-Phong lighting model [Bli77] over a photography-based texture mapping, as introduced by Catmull [Cat74].

Projections from 2D to 3D in texture mapping may cause artifacts in renderings. Distortions, discontinuities, and periodicity decrease the realism of liver graphics representation. Considering these issues, Neyret and Cani [NC99] proposed an algorithm to map a set of pattern-based texture samples with specific boundary conditions:

- Texture generation: a set of equilateral triangles represents texture patches which results from a generalized Worley texture synthesis [Wor96]. To compute these patches, the method subdivides the texture in a triangular grid. Every grid cell has a random noise value which defines a point in that region. Moreover, edges of adjacent patches have equaled values and derivative along their respective boundaries. Finally, each pixel receives a color computed from the distances to the 9 closest points in the grid, resulting in a generalized Voronoi Diagram.

- Texture mapping: the method presents a second mesh where 
its vertices are equally distributed points (considering geodesics distances). Therefore, they perform this mesh texture coordinates projection over the original geometric mesh.

In 2002, Neyret et al. [NHS02] argued that the liver surface has a wet look over cellular noise patterns. Therefore, they extended the previous technique considering a 3-texture multilayered texture mapping as follows:

- Skin: a diffuse procedural texture computed according to the mentioned Neyret and Cani [NC99] work.

- Reflections: this layer is a generalized reflection map [MH84] where a smooth ring (expressed through a 2D radial ramp) simulates the surgery shaped light-source reflection over the organ surface.

- Reactions: a texture built in real-time according to user interactions. Represents effects such as blood drops, cauterization, and whitening (which is caused by pressure over the organ surface). A set of disks with dynamic color, intensity, and opacity are used to represent the effects' appearance.

For an accurate blending of the textures, they applied Phong Shading, assessing the Blinn-Phong lighting model [Bli77] for each fragment of the image. However, these techniques may produce plastic-like surfaces, as denoted by Elhelw et al. [ELDzY04].

Hao et al. [HLX* 09 ] introduced the liver mucous layer modeling by rendering disrupted specular reflections and subsurface scattering in real-time. This technique extended the Blinn-Phong lighting model, where the diffuse component was computed by 3D model normal $N$ while the specularity used another normal $N^{\prime}$ from a Bump Map generated by Perlin noise [Per85]. For subsurface scattering, they applied the Jensen et al. [JMLH01] multiple scattering approximation. This model is a function of the distance between the incident and outgoing points where the light travels through a semi-translucent surface, or in other words, a BSSDF (Bidirectional Surface Scattering Reflectance Distribution Function).

Liu et al. [LWHL12] extended the previous method presenting two techniques in order to simulate the liver's diffuse texture. Standard procedural noise textures [Per85] simulate the organ tissue pattern together with an example-based texture [Ash01], where a real pattern is continuously replicated in a larger image according to their pixels neighborhood similarity.

The simulation of the liver's tissue diffuse texture with procedural methods, as presented by Liu and his colleagues, adds natural look for this 3D organ overall rendering. However, Nunes et al. [NMCW17] argued that the rendering of organic tissues requires lighting models that fit the actual material optical properties. In this context, they introduced a method to estimate the BRDF of in vivo liver using laparoscopy videos, rendered within a global illumination approach.

Laparoscopy is a Minimally Invasive Surgery where a camera with embedded light-source (laparoscope device) inside the patient provides the real-time visualization for the surgeons. They advanced the BRDF sampling for the organic live tissue by using a second laparoscope to capture footage with a wider range of cameralight combinations. This increased the BRDF coverage by one order of magnitude, when compared with methods which use only collinear sampling for internal organs as in [CDS*04] and [MB12], adding retroreflective and non-retroreflective samples. It is worth noting that, regarding the BRDF geometry $\left[\mathrm{NRH}^{*} 92\right]$, they defined the BRDF coverage as the sampling area integrated over the multiple hemispheres of light input and output directions. Once the BRDF data was estimated, they fitted the parameters to the isotropic version of the Ashikhmin lighting model [AS00] which, according to Ngan [NDM05], was a suitable choice for representing materials specular behaviors among physically plausible models. Finally, Nunes and his colleagues provided a physically based rendering of the liver according to their method, as depicted in Fig. 16-c.

According to the above review, liver rendering gradually advanced from shape-only approaches to Physically Based material modeling.

\section{Muscle Tissue}

Muscle tissues have three different types which are classified according to their functions, structures, and locations. Skeletal muscles provide voluntary movements of the skeleton through motile forces ${ }^{1}$ produced by contracting their striations - stripe-like visual features found in skeletal muscle, and transverse bands. The second class, the smooth muscle, produces motile forces in organs walls for activities like peristalsis ${ }^{2}$, parturition ${ }^{3}$ and so on. Cardiac muscle is a striated tissue controlled by the autonomic nervous system which forms the myocardium of the heart wall [ONN13]. The cells of the muscle tissue are, in fact, fibers containing filaments of the myosin and actin proteins. They work as parallel arrays which interact with each other causing muscle fibers contraction or shortening. For computer graphics, the muscle tissue motile forces become an important theme for research mainly for haptics in virtual environments. For our purposes, the appearance of muscle tissue is important for modeling and rendering of the eyes.

\subsection{Eyes}

In 2014, Ruhland et al. [RAB*14] presented a state-of-the-art report on the many issues associated with computer graphics research on eyes, including modeling, animation, and rendering. Here, we focus only on the appearance modeling and update the previous report with the more recent approaches. Eyes are the second most common topic of research in living human tissue, second only to skin.

Eyes are complex structures composed of many parts with all 4 types of tissue present. For appearance modeling, usually only the eyeball is relevant, illustrated in Fig. 12. The white part - called sclera - is made of connective tissue and the iris of muscle tissue. Since the iris is the most recognizable part, we classified the eyes as muscle tissue. Also part of the iris is the rounded aperture called pupil, where light passes through the lenses to reach the retina, made of receptor cells.

Motility is the ability to move spontaneously and actively, consuming energy in the process.

Peristalsis is a radially symmetrical contraction and relaxation of muscles that propagates in a wave down a tube, such as the digestive tube. Parturition is the action of giving birth. 


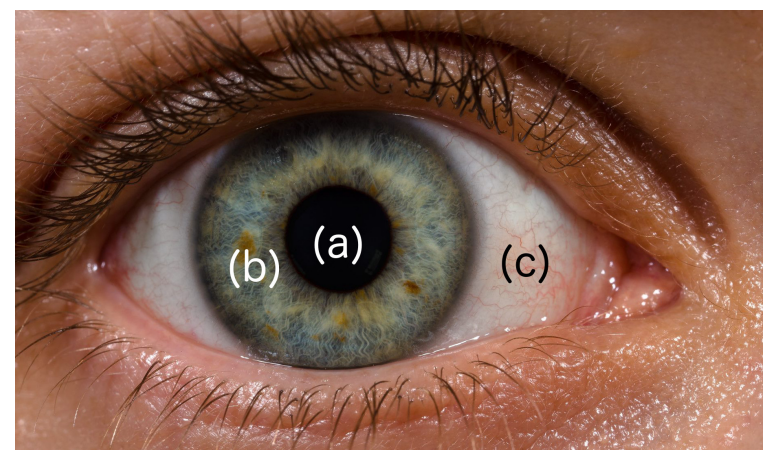

The earliest eye rendering was presented in 1994 by Sagar and colleagues [SBMH94]. They presented a virtual reality surgical simulator targeted for robotic eye surgery. All visible components of the eye were modeled, including the eyelashes. The sclera was rendered as simple Gouraud shaded polygons. For the iris, they developed two possibilities: a procedural model or texture mapping, and their use combined or alone was determined by the required performance of the tool. The iris procedural model was composed by two layers of individually shaded polygons to account for the many iris fibers. Retinal blood vessels, partially visible through the sclera, were modeled as a fractal tree [Opp86].

Arguably, the iris has been the most targeted part of the human eye in appearance modeling. Lefohn et al. [LBS*03] used inspiration from the making of eye protheses to drive their model for eye and iris synthesis. Ocularists are professionals who specialize in making prosthetic eyes, using a laborious process perfected over years, with very convincing visual quality. The artificial eye is made through a detailed process of hand painting a large number of semitransparent overlapping layers. Each layer will bring more depth and detail to the overall result. The approach taken by the authors uses digitized copies of these semitransparent layers as texture maps and raytrace them over a collection of right circular cones representing the geometry. Area lights are added as environment maps, increasing the final visual quality.

The only biophysical model for iris synthesis was introduced by Lam and Baranoski in 2006 [WLVB06]. Using knowledge about the iris constitution and eye morphology, they developed a three layer model called ILIT, for Iridal Light Transport Model. Light rays reach and traverse iridal tissues following optical properties according to stochastic processes controlling the next state of a ray, reflected or refracted. The model is completed with scattering and absorption events affecting ray direction and termination. The whole process is implemented using a Monte Carlo approach and therefore is not amenable to realtime simulations. A set of biophysical meaningful parameters allow great flexibility and control over the results. The radiometric outputs of the model were validated against actual measured iridal data, showing a general quantitative agreement.

The high computational cost of the ILIT model motivated the search for faster alternatives. François and colleagues presented in 2009 [FGBB09] a reasonably complex pipeline of operations to render clones of real eyes in realtime. First, from two iris pho- tographs - with and without reflections - they extract the 3D shape of the iris. This step includes unrefracting the iris image due to the cornea curvature. Next, they estimate scattering parameters for this particular geometry using an iterative method based on comparisons between images of the real and the virtual irises. Finally, the virtual iris is rendered at interactive rates using Subsurface Texture Mapping [FPBB08] augmented with a refraction and caustic functions.

More recently, Bérard and colleagues [BBN* 14$]$ presented a complete and complex framework for capturing both eye geometry and appearance, similar to [FGBB09] in the goal of eye cloning for digital doubles. Their approach included a special hardware setup with cameras, flashes, and LED lights for proper data acquisition, capturing eyes from real actors. Following the image acquisition step, first the sclera, and then cornea and iris are modeled. The final renderings present a distinct organic high quality, however, it takes around 4 hours for the reconstruction and rendering process to take place. A visual comparison between synthetic eyes rendered with François's technique (2009) and Bérard's technique (2014) is given in Fig. 13. Also, as presented, there is no possibility for customization of the acquired geometry and texture.

As a method to efficiently provide the rendering of customized eyes, Bérard et al. [BBGB16] proposed a strategy for leveraging the reconstructions of the previous work merging samples of 30 subjects according to their properties variation. In fact, they presented a parametric model where the shape, iris, and sclera where individually computed over the database samples to approximate arbitrary eyes appearance.

The geometry of the sclera is computed by customizing a geometric proxy for the eyeball using an image-based approach. Sclera texture is computed from the captured images augmented with texture synthesis techniques [EL99]. The cornea is modeled with an optimization approach applied to the control points of a B-Spline representing the cornea, using a set of reflection, refraction and position constraints from the images. The 3D iris is reconstructed together with the pupil, also using optimization to find the final shape from an initial similar shape. Due to the quality of image capture, iris texture maps are used to render the final images.
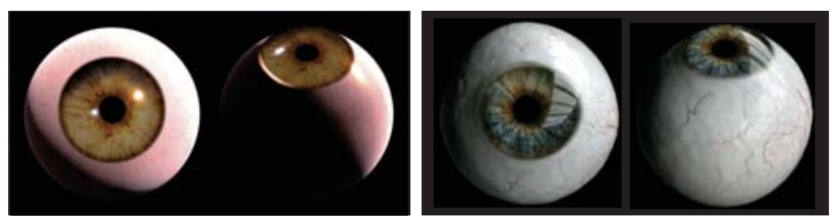

Figure 13: Visual comparison between results for eye rendering from [FGBB09] on the left and $\left[B B N^{*} 14\right]$ on the right. Source: original papers.

One possible drawback of models from first principles is their usually high computational cost. This issue is addressed on a recent paper by Kravchenko et al. [KBC*17a]. The iridal light transport model presented in [WLVB06] was reengineered such that it is possible to run simulations in realtime. By using CUDA [CBM*08] coupled with careful evaluation of the key parameters used in the 
simulation, they were able to achieve interactive rates. The main idea was to downsample the number of spectral samples on the spectral curves and the number of rays used in the Monte Carlo simulation. To fine-tune how much downsampling was adequate, they used a device independent CIE metric [MEO94] which states that below a certain threshold, a human observer cannot distinguish between similar images. This successful strategy could be used with similar computing intensive models, easing the adoption of specialized predictive rendering models in realtime applications such as games.

\section{Nervous Tissue}

The nervous tissue composes the central (CNS) and peripheral (PNS) nervous systems based on two types of cells: supporting cells (glia) and nerve cells (neurons). Neurons are a highly specialized cell which generates nervous impulses and transmits them to other neurons and organs. This conductivity is not present in glial cells since their function is mainly to support and protect neurons. Some CNS structures present arterial and venous vessels transversing the nervous tissue through the cerebrospinal fluid (a colorless fluid derived from blood plasma). The amount of myelin (a fatty white substance which is an electrical insulator) in a CNS tissue controls its appearance aspect, for example, as in the brain gray matter [ONN13, Mar15].

\subsection{Brain}

For computer graphics purposes, the brain is the only interesting organ made of nervous tissue, being the largest and most complex mass of nervous tissue in our bodies [Mar15]. The cerebrum is the largest part of the brain and is composed of right and left hemispheres. It is the cerebrum that is usually exposed when a segment of skull is removed during a neurosurgery procedure. The surface of the cerebrum has a folded appearance called the cortex that has a gray-brown color. The surface membrane of the brain also contains a network of blood vessels of various sizes and structure.

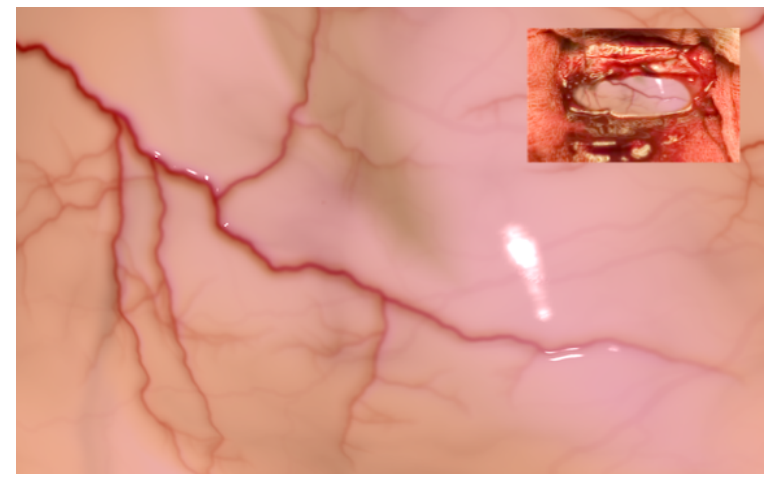

Figure 14: The final rendering of the living brain surface using the preferred BRDF and color from calibrated operating theater photographs. Subsurface scattering has been applied and the veins are procedurally generated. The inset represents the brain surface visible following a craniotomy. Source: [aCJB*12])
One approach to rendering the brain surface involves calculating a BRDF to model how light reflects on the surface of the brain [aCWJ*11, aCJB*12]. The final result (see Fig. 14) uses color information obtained from photographs taken during a neurosurgery procedure and a BRDF measured from a substitute material as the process of calculating a calibrated BRDF was too obtrusive to be obtained during the surgery being performed on the patient. Several different parameters for the brain BRDF were considered including measurements obtained from a cadaver brain. The results were validated by neurosurgeons and anatomy experts and it is interesting to note that the cadaver measurements were not seen as realistic. In fact, animal meat with water flowing over it produced the best results for the BRDF model. Lamb steak was selected. The final rendering is also enhanced by the use of sub-surface scattering and the brain surface is overlaid with a network of procedurallygenerated veins. It has been implemented on the GPU for real-time rendering.

\section{General Approaches}

Here we review research that was not clearly part of the previous groups because they address more than one type of tissue or have a general approach useful for all types. For instance, Sunguroff and Greenberg [SG78] established the standard pipeline for rendering organic structures based on CT-Scan images. In their method, segmented images of the organs contour were fitted by B-Splines curves, producing a 3D geometry. The material appearance was computed by the Blinn-Phong [Bli77] reflection model, which results in a smooth, colored, and translucent surface. This method allows the $3 \mathrm{D}$ visualization of the human organs, however, no specific material property was taken into account. Similarly, Robb [Rob99] used the Visible Human project [Ack98] data as input to improve the organs geometric reconstruction. However, the organic material representation was reduced to the Lambert reflection model over interpolated textures from the same dataset, which neglects specular reflections but improves the rendering level of detail.

Texture mapping represents a powerful method for depicting complex structures commonly found on organ surfaces. However, due to anatomical constraints, this approach requires the manual definition of texture coordinates. To optimize this task, Szekely et al. [SBD*00] used 3D textures which were synthesized from specific organs tissue patches mapped over arbitrary shapes. When required, procedural texture methods were combined to simulate structures on the organ surface as blood vessels (computed by a generalized L-system [PL96]), pigmentation (noise functions [Per85]), and follicles (random points along the texture). In order to remove plastic-like artifacts, they extended the Blinn-Phong model [Bli77]. Their strategy was to boost highlights where the standard equation computes its maximum value. Recently, Berndt and colleagues successfully applied the Visible Human dataset as a 3D texture to render any tissue, from bone to blood, while cutting through the body in real time in a simulated surgery [BTM17].

As an alternative method to avoid the plastic-like appearance in rendering, ElHelw et al. [ELDzY04] applied 2D maps computed by Perlin noise functions [Per85] to represent tissues roughness and their mucous layer. Therefore, a normal map disrupts the specular component computing, while an additional texture map produces 
color for blending with the surface original color, in realtime (see Fig. 15). In 2005, this technique was evaluated by a group of naive volunteers and experts surgeons in a bronchoscopy surgery context $\left[E^{*} 05\right]$. The visual results show improvements when compared with standard Phong-like models for organic tissue representation.
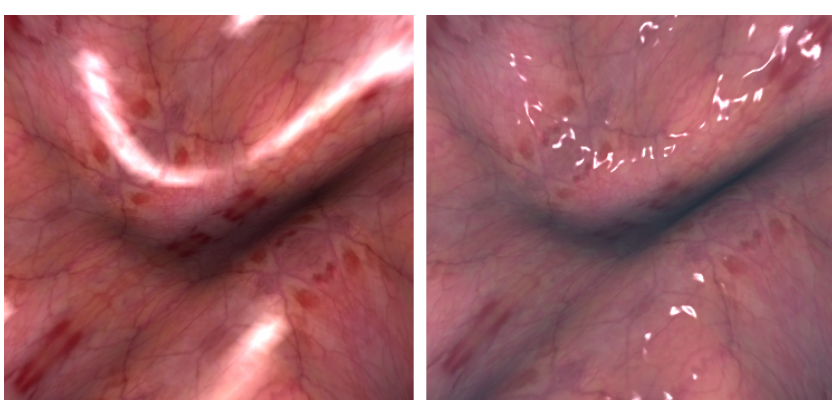

Figure 15: A standard texture rendering with Blinn-Phong lighting model (left) and a result computed by roughness and mucous layer color mapping (right). Source: [ $\left.E^{*} 05\right]$.

In the context of applications for modeling the appearance of organic tissue, surgery simulators scenes represent a challenge due to the different types of materials present, such as membranes and fluids, together with interactive surgery tools. Lim and colleagues [LJD07] approached this issue in their image-based rendering method for virtual surgery background reconstruction from actual MIS videos. They applied image mosaicing and view-dependent texture mapping to enhance a laparoscopy simulator rendering considering only local material-light interactions. Qian et al. [QBY*15] claim that physics-based methods are alternatives for computing accurate graphics representation in such medical applications. As expressed by Kajiya [Kaj86], physically plausible approaches integrate the lightmaterial interaction for the whole 3D scene. Therefore, Qian and colleagues presented an entire Physically Based rendering pipeline for a laparoscopy simulator. First, a texture represented the local diffuse reflection according to its correspondent normal map, attenuated by Burley's diffuse approximation [Bur12]. The environment diffuse lighting was precomputed by a set of nine Spherical Harmonic basis function [RH01]. These frequency space functions capture the diffuse reflection of an environment map for an entire hemisphere. For the specular term, the Cook-Torrance [CT82] model was sampled on the environment map, also attenuated by the Spherical Harmonics lighting, as defined by Kasyan [Kas13]. Finally, the diffuse and specular terms ratio was controlled by the Tsai and Shih [TS06] Fresnel approximation. As detailed in Qian et al. [QBY* 16], this method allows preserving physical constraints as energy conservation while rendering organic material in a global lighting context.

\section{Conclusions}

The modeling of natural phenomena has a long tradition in computer graphics research [BGM $\left.{ }^{*} 83\right]$. After approximately 40 years, we now have an incipient but also substantial body of research on the inner human body and its many tissues and organs, as we briefly show in this survey.
Our review has presented a taxonomy for computer graphics modeling of living human tissue. This taxonomy used the four types of human tissues as the main classifier. We reviewed the main approaches published to date concerned with appearance modeling for this class of organic materials. We have also related the human tissues biology to the light-material interaction graphics representation adopted in those works. A brief visual summary of advances achieved so far in computer graphics research is given in Fig. 16. From top to bottom, we show the progress on rendering for the skin, the liver, and the eye. From left to right we notice the improvements in the visual quality provided by Physically Based and Biophysically Based approaches over earlier Functional approaches. The modeling sophistication of Biophysically Based approaches, although in principle more computationally expensive, allow increasing levels of visual quality, since the realism is intrinsic to the model.

When we consider the average year of all publications in each modeling class presented in Section 3, we notice that the average year of all Functional models is 2001; Biophysically Inspired models is 2008; Physically Based models is 2011; and Biophysically Based models is 2012. From these numbers we can argue a trend where Biophysically Based approaches will concentrate most, if not all, future research on appearance modeling of live human tissues. This trend is illustrated in Figure 17, where we group the reviewed papers by modeling classes and year of publication.

The human body is an immense source of open problems if we ever want to reach the same level of maturity that we now have for manufactured materials. At the same time, the challenges are seemingly greater since organic material is in general inherently more complex than non-organic materials. We have seen detailed models for the skin on the face, but not yet for other body parts where skin is significantly different. How about light interacting with the pigments deposited on inner layers of tattooed skin? The variety of skin colors across all ethnic populations and the variation of hair density on skin provide additional rendering problems. In Osteology, just recently we have seen models for teeth (Sec. 6) but we still do not have a single model for bones in general. Such gaps exist in most of the areas considered above, i.e., there is better literature about tissues with easier access for sampling experiments or stable appearance (at least for some hours).

Maybe the past and current applications have not placed a high enough demand for live human tissue models, while game and movie industries are rather pragmatic, accepting that something is visually correct if it is perceived as plausible.

Now, in a glimpse of future creativity, let us discuss about the possibilities once more powerful models are available. Certainly, more realistic skin and eyes will benefit games and movies. They will also improve user experience in immersive virtual reality with better virtual human characters. It seems, however, that Biophysics based models of living internal human parts will attract a wider audience in health applications. Today's functional models already impact the way surgeons practice and plan surgery [BCHSS99, HSD10, BMPG11,HWCH12,BPBG13,UF15,BTM17]. Virtual patients are an alternative to cadaver or animal models for the learning of anatomy and surgical techniques. With the availability of Biophysical based models of more tissues and organs, virtual patients 

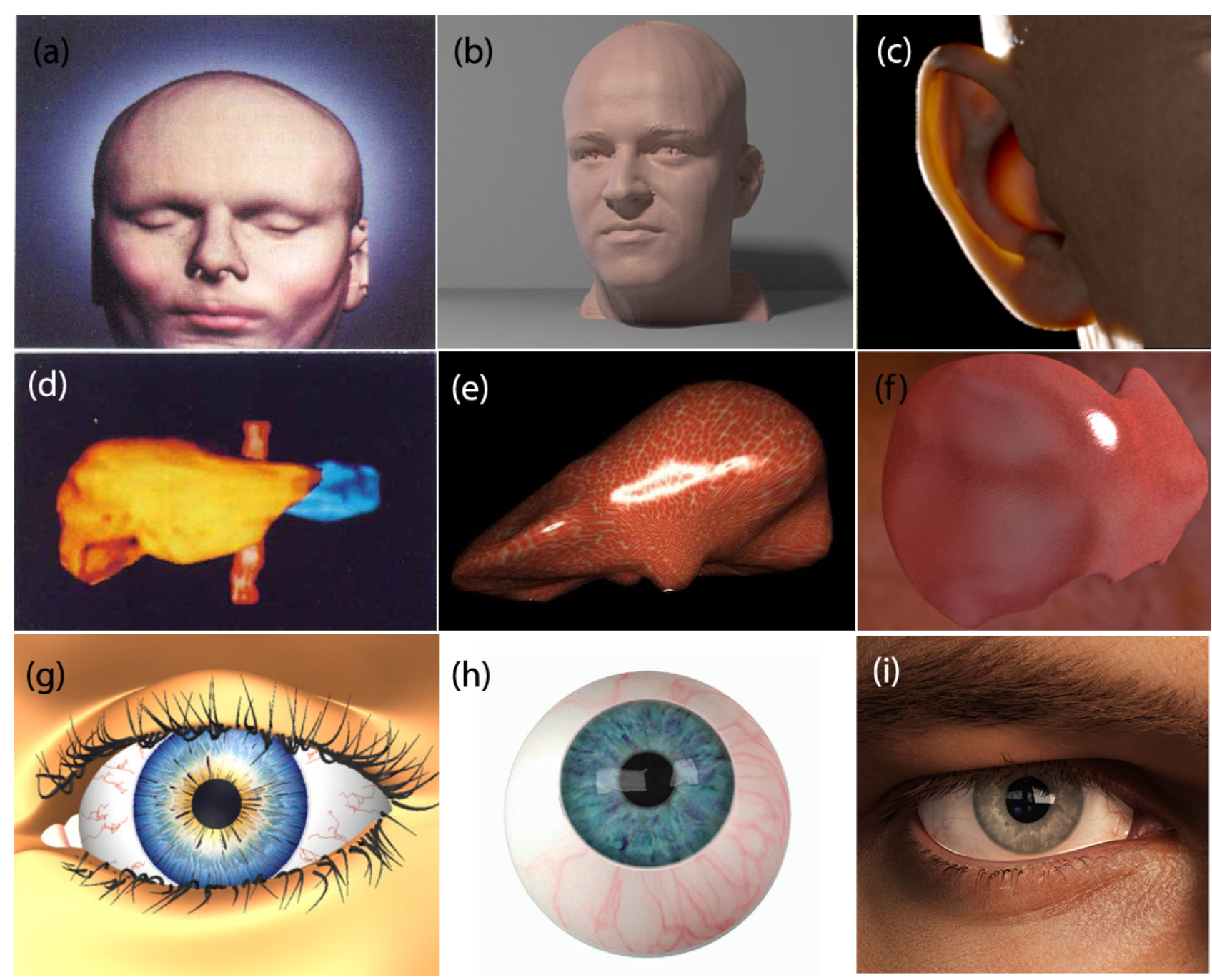

(h)

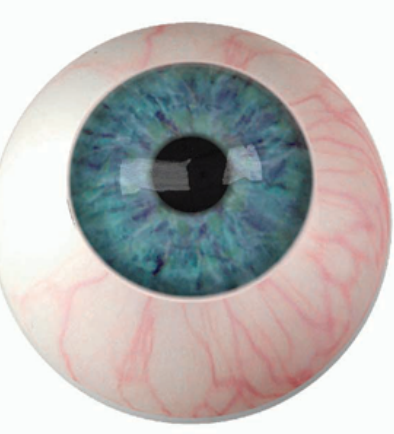

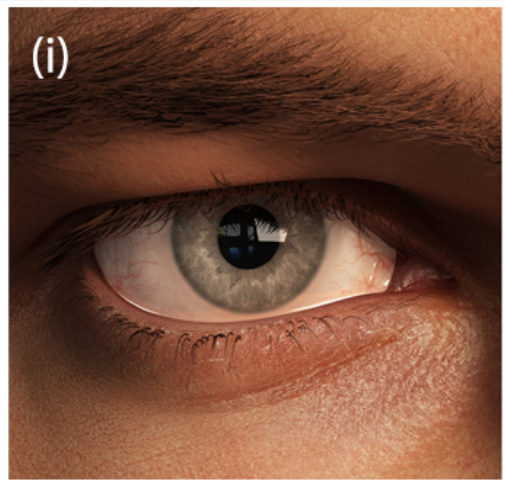

Figure 16: Examples of results for different human tissues and modeling classes. Top: (a) skin modeling from [HK93] (Biophysically Inspired), (b) [MWL 99] (Physically Based), and (c) [IGAJG15] (Biophysically Based). Middle: (d) liver tissue modeling from [KMKK88] (Functional), (e) [NHSO2] (Biophysically Inspired), and (f) [NMCW17] (Physically Based). Bottom: ( $g$ ) eyes tissue modeling from [SBMH94] (Functional), (h) [LBS* 03] (Biophysically Inspired), and (i) [KBC ${ }^{*}$ 17b] (Biophysically Based). Source: adapted from the original papers.

could be an affordable and ethical replacement over current practices. These would also likely be configurable, where pathology, age, and health condition could be represented in the model and would faithfully show the accurate appearance of symptoms of a specific patient.Using improved material models will also support soft tissue modeling and the use of haptic devices in medical training simulators [CMJ11].

As a general trend, we can expect more solutions addressing the dynamic nature and individuality of tissues, together with external factors. For virtual surgery simulations, we need models able to vary the appearance according to demographic data such as age and general health conditions (smoker, non-smoker, overweight, etc). Once we know how to build biophysical models with meaningful parameters, predictive rendering becomes a reality and our knowl- edge can be used beyond just picture making, as already pointed out by Greenberg and colleagues in 1997 [GAL ${ }^{*} 97$ ].

\section{References}

\section{HVFFTPS 12}

Alkawaz M. H., Basori A. H., Mohd Hashim S. Z.: Oxygenation absorption and light scattering driven facial animation of natural virtual human. Multimedia Tools and Applications (2016), $1-37$.

ap Cenydd L., John N. W., Bloj M., Walter A., Phillips N. I.: Visualizing the surface of a living human brain. IEEE Computer Graphics and Applications 32, 2 (2012), 55-65. 


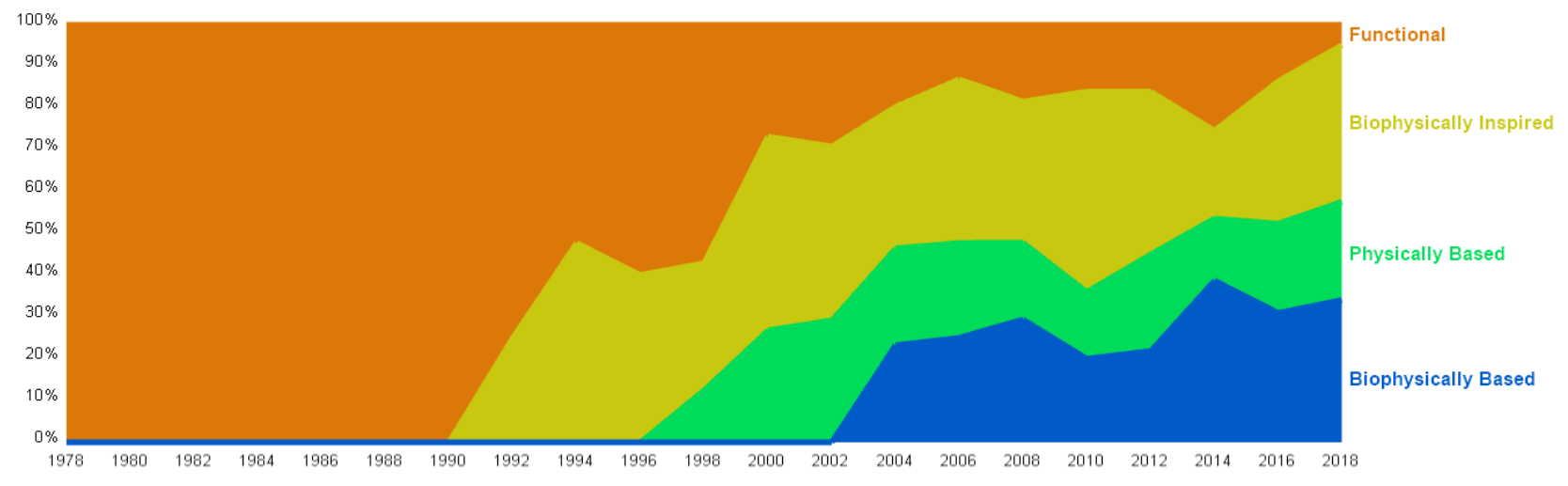

Figure 17: Appearance modeling classes distribution throughout 40 years of computer graphics research considering the surveyed papers.

Ackerman M. J.: The visible human project. Proceedings of the IEEE 86, 3 (Mar 1998), 504-511.

ap Cenydd L., Walter A., John N. W., Bloj M., Phillips N. I.: Realistic visualization of living brain tissue. In The 18th Medicine Meets Virtual Reality (2011), pp. 105-111.

A. FELlner F.: Introducing cinematic rendering: A novel technique for post-processing medical imaging data. Journal of Biomedical Science and Engineering 09 (01 2016), 170-175.

Aggarwal R., Mytton O. T., Derbrew M., Hananel D., Heydenburg M., Issenberg B., MacAulay C., Mancini M. E., Morimoto T., Soper N., Ziv A., ReZnick R.: Training and simulation for patient safety. BMJ Quality \& Safety 19, Suppl 2 (2010), i34-i43.

Alkawaz M. H., Mohamad D., Saba T., BAsori A. H., Rehman

A.: The correlation between blood oxygenation effects and human emotion towards facial skin colour of virtual human. $3 D$ Research 6, 2 (2015), 13.

ANDERSON R., PARRISH J.: Optical properties of human skin. In The Science of Photomedicine (N.Y., USA, 1982), Regan J., Parrish J., (Eds.), Plenun Press, pp. 147-194.

ASHIKHMIn M., SHIRLEY P.: An anisotropic phong brdf model. Journal of Graphics Tools 5, 2 (2000), 25-32.

Alotaibi S., Smith W. A. P.: A biophysical 3d morphable model of face appearance. In 2017 IEEE International Conference on Computer Vision Workshops (ICCVW) (Oct 2017), pp. 824-832.

AshiKhmin M.: Synthesizing natural textures. In Proceedings of the 2001 Symposium on Interactive 3D Graphics (New York, NY, USA, 2001), I3D ’01, pp. 217-226.

BÉrARd P., Bradley D., Gross M., BeEler T.: Lightweight eye capture using a parametric model. ACM Trans. Graph. 35, 4 (July 2016), 117:1-117:12.

Bérard P., Bradley D., Nitti M., BeEler T., Gross M. H.: High-quality capture of eyes. ACM Trans. Graph. 33, 6 (2014), 223-1.
Basdogan C., Chih-HaO, Srinivasan M. A., SRinivasan A. A.: Simulation of tissue cutting and bleeding for laparoscopic surgery using auxiliary surfaces. In In Medicine Meets Virtual Reality (1999), IOS Press, pp. 38-44.

Bashkatov A., Genina E., Kochubey V., Tuchin V.: Optical properties of human skin, subcutaneous and mucous tissues in the wavelength range from 400 to $2000 \mathrm{~nm}$. Journal of Physics D: Applied Physics 38 (2005), 2543-2555.

Blinn J., Gomez J., Max N., Reeves W., Chairman-Csuri C. A.: The simulation of natural phenomena (panel session). In ACM SIGGRAPH Computer Graphics (1983), vol. 17, ACM, pp. 137139.

Bashkatov A., Genina E., Tuchin V.: Optical properties of skin, subcutaneous, and muscle tissues: A review. Journal of Innovative Optical Health Sciences 4, 11 (2011), 9-38.

BARANOSKI G., KRISHNASWAMY A.: Light \& Skin Interactions: Simulations for Computer Graphics Applications. Morgan Kaufmann/Elsevier, Burlington, MA, USA, 2010.

BLINN J. F.: Models of light reflection for computer synthesized pictures. SIGGRAPH Comput. Graph. 11, 2 (July 1977), 192-198.

Borgeat L., Massicotte P., Poirier G., Godin G.: Layered surface fluid simulation for surgical training. In Medical Image Computing and Computer-Assisted Intervention - MICCAI 2011, Fichtinger G., Martel A., Peters T., (Eds.). Springer Berlin Heidelberg, 2011, pp. 323-330.

Boisvert J., Poirier G., Borgeat L., Godin G.: Real-time blood circulation and bleeding model for surgical training. IEEE Transactions on Biomedical Engineering 60, 4 (April 2013), 10131022.

Berndt I., TORCHELSEn R., MACIEL A.: Efficient surgical cutting with position-based dynamics. IEEE Computer Graphics and Applications 38, 3 (May 2017), 24-31.

BURLEY B.: Physically-based shading at disney. In SIGGRAPH: ACM SIGGRAPH 2012 Courses, ACM., 2012. 
BUTLER W.: Absorption spectroscopy in vivo: theory and application. Annu. Rev. Plant Phys. 15 (1964), 451-470.

Baranoski G., Van Leeuwen S., Chen T.: Elucidating the biophysical processes responsible for the chromatic attributes of peripheral cyanosis. In 39th Annual International Conference of the IEEE Engineering in Medicine and Biology Society (EMBC) (2017), IEEE, pp. 90-95.

Barros R. S., Walter M.: Synthesis of human skin pigmentation disorders. Computer Graphics Forum 36, 1 (2017), 330-344.

Bachofen D., Zátonyi J., Harders M., SzéKely G., Fruh P., ThALER M.: Enhancing the visual realism of hysteroscopy simulation. Studies in health technology and informatics 119 (2005), 31.

Catmull E. E.: A Subdivision Algorithm for Computer Display of Curved Surfaces. PhD thesis, 1974. AAI7504786.

Chen T. F., Baranoski G. V. G., Kimmel B. W., Miranda E.: Hyperspectral modeling of skin appearance. ACM Trans. Graph. 34, 3 (may 2015), 31:1-31:14.

Che S., Boyer M., Meng J., Tarjan D., Sheaffer J. W., SKADRON K.: A performance study of general-purpose applications on graphics processors using cuda. Journal of parallel and distributed computing 68, 10 (2008), 1370-1380.

Cotton S., Claridge E., Hall P.: A skin imaging method based on a colour formation model and its application to the diagnosis of pigmented skin lesions. 49-52.

Chung A. J., Deligianni F., Shah P., Wells A., Zhong YANG G.: Enhancement of visual realism with brdf for patient specific bronchoscopy simulation. In Proceedings of the Lecture Notes in Computer Science (2004), SpringerVerlag, pp. 486-493.

Chung A., Deligianni F., Shah P., Wells A., Yang G.Z.: Patient-specific bronchoscopy visualization through brdf estimation and disocclusion correction. Medical Imaging, IEEE Transactions on 25, 4 (april 2006), 503-513.

Choi T., Ghan S., Chin S.: Biological property-based artificial scar synthesis using inverse lighting. Multimedia Systems (Jul 2017).

CHEN T.: On the Modelling of Hyperspectral Light and Skin Interactions and the Simulation of Skin Appearance Changes Due to Tanning. PhD thesis, Natural Phenomena Simulation Group, David R. Cheriton School of Computer Science, University of Waterloo, Canada, December 2015.

Chan S., Li P., Locketz G., Salisbury K., Blevins N. H.: High-fidelity haptic and visual rendering for patient-specific simulation of temporal bone surgery. Computer Assisted Surgery 21, 1 (2016), 85-101.

Correa C., MA K. L.: The occlusion spectrum for volume classification and visualization. IEEE Transactions on Visualization and Computer Graphics 15, 6 (Nov 2009), 1465-1472.

Coles T., Meglan D., John N. W.: The role of haptics in medical training simulators: a survey of the state of the art. Haptics, IEEE Transactions on 4, 1 (2011), 51-66.
Chen B., Stamnes K., Stamnes J.: Validity of the diffusion approximation in bio-optical imaging. Applied Optics 40, 34 (2001), $6356-6336$

COOK R. L., TORRANCE K. E.: A reflectance model for computer graphics. ACM Trans. Graph. 1, 1 (Jan. 1982), 7-24.

Drebin R. A., CARPENTER L., HanRahan P.: Volume rendering. SIGGRAPH Comput. Graph. 22, 4 (June 1988), 65-74.

De GraAfF K. V.: Human Anatomy, 4th ed. W. C. Brown Publishers, Dubuque, IO, USA, 1995.

Dick C., Georgit J., Burgkart R., Westermann R.: Stress tensor field visualization for implant planning in orthopedics. IEEE Transactions on Visualization and Computer Graphics 15, 6 (Nov 2009), 1399-1406.

DORSEY J., HANRAHAN P.: Modeling and rendering of metallic patinas. In The 23rd Annual Conference on Computer Graphics and Interactive Techniques (SIGGRAPH '96) (New York, NY, USA, 1996), ACM, ACM, p. 387-396.

Dappa E., Higashigaito K., Fornaro J., LeschKa S., WilDERMUTH S., ALKADHI H.: Cinematic rendering - an alternative to volume rendering for $3 \mathrm{~d}$ computed tomography imaging. Insights into Imaging 7, 6 (Dec 2016), 849-856.

Debevec P., Hawkins T., TChou C., Duiker H.-P., SARokin W., SAGAR M.: Acquiring the reflectance field of a human face. In Proceedings of the 27th Annual Conference on Computer Graphics and Interactive Techniques (2000), SIGGRAPH '00, pp. 145-156.

D'EON E., IRVING G.: A quantized-diffusion model for rendering translucent materials. ACM Transactions on Graphics 30, 4 (2011), $56: 1-13$.

DONNER C., JENSEN H.: Light diffusion in multi-layered translucent materials. ACM Transactions on Graphics 24, 3 (July 2005), 1032-1039.

Donner C., Jensen H.: A spectral bssrdf for shading human skin. In Rendering Techniques 2006: 17th Eurographics Workshop on Rendering (June 2006), pp. 409-418.

D'Eon E., LuebKe D., Enderton E.: Efficient rendering of human skin. In Rendering Techniques 2007: 18th Eurographics Workshop on Rendering (June 2007), pp. 147-157.

Dorsey J., Rushmeier H., Sillion F.: Digital Modeling of Material Appearance. Morgan Kaufmann, 2008.

DONNER C., WEYRICH T., D'EON E., RAMAMOORTHI R., RUSINKIEWICZ S.: A layered, heterogeneous reflectance model for acquiring and rendering human skin. ACM Transactions on Graphics 27, 5 (2008), 140:1-12

ElHelw M. A., ET AL.: Photo-realistic tissue reflectance modelling for minimally invasive surgical simulation. In Medical Image Computing and Computer-Assisted Intervention - Proceedings, Part I, Duncan J. S., Gerig G., (Eds.). Springer Berlin Heidelberg, Berlin, Heidelberg, 2005, pp. 868-875.

Estai M., BUNT S.: Best teaching practices in anatomy education: A critical review. Annals of Anatomy-Anatomischer Anzeiger 208 (2016), 151-157. 
Elbischger P. J., CACho R., Bischof H., Holzapfel G. A.: Modeling and characterizing collagen fiber bundles. In $3 r d$ IEEE International Symposium on Biomedical Imaging: Nano to Macro, 2006. (April 2006), pp. 1280-1283.

EFros A. A., LEUNG T. K.: Texture synthesis by non-parametric sampling. In Proceedings of the Seventh IEEE International Conference on Computer Vision (1999), vol. 2, pp. 1033-1038 vol.2.

Evgeniou E., Loizou P.: Simulation-based surgical education. ANZ Journal of Surgery 83, 9 (2013), 619-623.

Elhelw M. A., Lo B. P., DARZI A., ZhONG YANG G.: Realtime photo-realistic rendering for surgical simulations with graphics hardware. In Proceedings of the Medical Imaging and Augmented Reality (2004), pp. 346-352.

FERWERDA J. A.: Three varieties of realism in computer graphics. In Proceedings of the SPIE Human Vision and Electronic Imaging (2003), pp. 290-297.

Francois G., Gautron P., Breton G., Bouatouch K.: Imagebased modeling of the human eye. IEEE Transactions on Visualization and Computer graphics 15, 5 (2009), 815-827.

Fried D., Glena R., Featherstone J., Seka W.: Nature of light scattering in dental enamel and dentin at visible and nearinfrared wavelengths. Applied Optics 34, 7 (1995), 1278-1285.

Frisvad J. R., HACHISUKa T., KJELdSEN T. K.: Directional dipole model for subsurface scattering. ACM Trans. Graph. 34, 1 (Dec. 2014), 5:1-5:12.

FeEss S., Kurfiss K., Michels D. L.: Accurate simulation of wound healing and skin deformation. In Proceedings of ACM SIGGRAPH / Eurographics Symposium on Computer Animation (2016).

Francois G., Pattanaik S., Bouatouch K., Breton G.: Subsurface texture mapping. IEEE Computer Graphics and Applications 28, 1 (Jan 2008), 34-42.

Greenberg D., Arvo J., Lafortune E., Torrance K., FerWerda J., Walter B., Trumbore B., Shirley P., Pattanaik S., Foo S.: A framework for realistic image synthesis. In SIGGRAPH, Annual Conference Series (1997), pp. 477-494.

GuYton A. C., Hall J. E.: Textbook of Medical Physiology. Elsevier, 2006.

Ghosh A., Hawkins T., Peers P., Frederiksen S., DebeveC P.: Practical modeling and acquisition of layered facial reflectance. ACM Transactions on Graphics 27, 5 (2008), 139:1-10.

Goncalves L. F. P.: Photorealistic modelling and rendering of $3 D$ human anatomy for medical training. Master's thesis, Universidade do Minho, Portugal, 2010.

GourAUD H.: Continuous shading of curved surfaces. IEEE Transactions on Computers C-20, 6 (June 1971), 623-629.

Graham P., Tunwattanapong B., Busch J., Yu X., Jones A., Debevec P., Ghosh A.: Measurement based synthesis of facial microgeometry. Computer Graphics Forum 32, 2 pt3 (2013), $335-344$.
Gkioulekas I., XiaO B., Zhao S., Adelson E. H., ZickLER T., BALA K.: Understanding the role of phase function in translucent appearance. ACM Trans. Graph. 32, 5 (Oct. 2013), 147:1-147:19.

Hunter R., HAROlD R.: The Measurement of Appearance, second ed. John Wiley \& Sons, New York, 1987.

Hielscher A., Jacques S., Wang L., TitTel F.: The influence of boundary conditions on the accuracy of diffusion theory in time-resolved reflectance spectroscopy of biological tissues. Physics in Medicine and Biology 40 (1995), 1957-1975.

HANRAHAN P., KRUEGER W.: Reflection from layered surfaces due to subsurface scattering. In Proceedings of the 20th Annual Conference on Computer Graphics and Interactive Techniques (New York, NY, USA, 1993), SIGGRAPH '93, ACM, pp. 165-174.

Hao A., Li S., Xu X., LiU X., Song F.: Real-time realistic rendering of tissue surface with mucous layer. Computer Science and Engineering, International Workshop on 01, undefined (2009), 302-306.

HaAse C. S., Meyer G. W.: Modeling pigmented materials for realistic image synthesis. ACM Trans. Graph. 11, 4 (Oct. 1992), 305-335.

Halic T., Sankaranarayanan G., De S.: Gpu-based efficient realistic techniques for bleeding and smoke generation in surgical simulators. The International Journal of Medical Robotics and Computer Assisted Surgery 6, 4 (2010), 431-443.

Hernández-Vaquero D., Fernández-Fairen M., TorresPerez A., SANTAMARÍA A.: Minimally invasive surgery versus conventional surgery. a review of the scientific evidence. Revista Española de Cirugía Ortopédica y Traumatología (English Edition) 56, 6 (2012), 444-458.

Huang J., Wu W., Chen H., Heng P. A.: Bleeding simulation in virtual abdominal surgery. In The 6th International Conference on Bioinformatics and Biomedical Engineering (2012).

Iglesias-Guitian J. A., Aliaga C., Jarabo A., Gutierrez D.: A Biophysically-Based Model of the Optical Properties of Skin Aging. Computer Graphics Forum (2015).

IgARASHI T., NISHINO K., NAYAR S.: The appearance of human skin: A survey. Found. Trends. Comput. Graph. Vis. 3 (January 2007), 1-95.

Ishimaru A.: Wave Propagation and Scattering in Random Media, 2 ed., vol. 1. IEEE Press, 1978.

Iwamoto N., Shum H. P. H., Yang L., Morishima S.: Multilayer lattice model for real-time dynamic character deformation. Computer Graphics Forum 34, 7 (2015), 99-109.

JACQUES S.: Skin optics. http://omlc.ogi.edu/news/jan98/skinoptics.html, 1998.

JACQUES S.: Optical properties of biological tissues: a review. Phys. Med. Biol. 58 (2013), R37-R61.

JENSEN H., BUHLER J.: A rapid hierarchical rendering technique for translucent materials. In SIGGRAPH, Annual Conference Series (July 2002), pp. 576-581. 
JENSEN H., Christensen P.: Efficient simulation of light transport in scenes with participating media using photon maps. In Proceedings of the 25th Annual Conference on Computer Graphics and Interactive Techniques (New York, NY, USA, 1998), SIGGRAPH '98, ACM, pp. 311-320.

Jung J. W., Meyer G., Delong R., Holmes B. N.: Rendering of human teeth and restorative biomaterials. In Color and Imaging Conference (2012), vol. 2012, Society for Imaging Science and Technology, pp. 170-176.

Jensen H. W., Marschner S. R., Levoy M., Hanrahan P.: A practical model for subsurface light transport. In Proceedings of the 28th Annual Conference on Computer Graphics and Interactive Techniques (New York, NY, USA, 2001), SIGGRAPH '01, ACM, pp. 511-518.

Jimenez J., Scully T., Barbosa N., Donner C., Alvarez X., Vieira T., Matts P., OrValho V., Gutierrez D., Weyrich T.: A practical appearance model for dynamic facial color. $A C M$ Transactions on Graphics 29, 6 (2010), 141:1-10.

JUNG J. W.: Rendering of Teeth and Dental Restorations using Robust Statistical Estimation Techniques. PhD thesis, UNIVERSITY OF MINNESOTA, 2016.

J. Welch A., J. C. VAn Gemert M.: Optical-thermal response of laser-irradiated tissue. Optical-Thermal Response of Laser-Irradiated Tissue by Ashley J. Welch and Martin J.C. van Gemert. Berlin: Springer, 2011. ISBN: 978-9-048-18830-7 (01 2011).

KAJIYA J. T.: The rendering equation. In SIGGRAPH '86 (New York, NY, USA, 1986), ACM, pp. 143-150.

KASYAN N.: Playing with real-time shadows. In SIGGRAPH: ACM SIGGRAPH 2013 Courses, ACM., 2013.

KRISHNASWAMY A., BARANOSKI G. V.: A biophysically-based spectral model of light interaction with human skin. Computer Graphics Forum 23, 3 (2004), 331-340.

Kimmel B. W., BARAnoski G. V. G.: A novel approach for simulating light interaction with particulate materials: application to the modeling of sand spectral properties. Optics Express 15, 15 (2007), 9755-9777.

Kravchenko B., Baranoski G. V., Chen T. F., Miranda E., VAN LEEUWEN S. R.: High-fidelity iridal light transport simulations at interactive rates. Computer Animation and Virtual Worlds 28, 3-4 (2017).

Kravchenko B., Baranoski G. V. G., Chen T. F., Miranda E., VAN LEEUWEN S. R.: High-fidelity iridal light transport simulations at interactive rates. Computer Animation and Virtual Worlds 28, 3-4 (2017), e1755-n/a.

KAJIYA J. T., KAY T. L.: Rendering fur with three dimensional textures. SIGGRAPH Comput. Graph. 23, 3 (July 1989), 271-280.

KhUngurn P., MARSCHNer S.: Azimuthal scattering from elliptical hair fibers. ACM Trans. Graph. 36, 2 (Apr. 2017), 13:1$13: 23$.

Kashiwagi T., Mitsutani N., Koizumi T., Kimura K.: Threedimensional demonstration of liver and spleen by a computer graphics technique. Acta Radiologica 29, 1 (1988), 27-31.
Kang Y., Park J., LeE J., Shim M., Kim M.: Compact modeling and plausible deformation of human lung anatomy with smooth surfaces. In Proceedings of the 29th International Conference on Computer Animation and Social Agents, CASA 2016, Geneva, Switzerland, May 23-25, 2016 (2016), pp. 21-28.

Klehm O., Rousselle F., Papas M., Bradley D., Hery C., Bickel B., JAROSZ W., BeEler T.: Recent Advances in Facial Appearance Capture. Computer Graphics Forum (2015).

Kerwin T., Shen H. W., STREDNEy D.: Enhancing realism of wet surfaces in temporal bone surgical simulation. IEEE Transactions on Visualization and Computer Graphics 15, 5 (Sept 2009), 747-758.

KhUngurn P., Schroeder D., ZhaO S., BALA K., MARSChNer S.: Matching real fabrics with micro-appearance models. ACM Trans. Graph. 35, 1 (dec 2015), 1:1-1:26.

Kollias N., SAYre R., Zeise L., Chedekel M.: Photoprotection by melanin. J. Photoch. Photobio. B. 9, 2 (1991), 135-60.

LATIMER P.: A wave-optics effect which enhances light absorption by chlorophyll in vivo. Photochem. Photobiol. 40, 2 (1984), 193 199.

Lefohn A., Budge B., Shirley P., Caruso R., Reinhard E.: An ocularist's approach to human iris synthesis. IEEE Computer Graphics and Applications 23, 6 (2003), 70-75.

LiU A. J., Dong Z., HašAn M., Marschner S.: Simulating the structure and texture of solid wood. ACM Trans. Graph. 35, 6 (2016).

LEVOY M.: Display of surfaces from volume data. IEEE Computer Graphics and Applications 8, 3 (May 1988), 29-37.

LARSEN C., Frisvad J., Jensen P., BÆrentzen J.: Real-time rendering of teeth with no preprocessing. Advances in Visual Computing (2012), 334-345.

LIM Y. J., JIN W., DE S.: On some recent advances in multimodal surgery simulation: A hybrid approach to surgical cutting and the use of video images for enhanced realism. Presence 16, 6 (Dec 2007), 563-583.

LiU X.-M., WANG S.-M., HaO A.-M., LiU H.: Realistic rendering of organ for surgery simulator. Computers and Mathematics with Applications 64, 5 (2012), 721 - 728. Advanced Technologies in Computer, Consumer and Control.

MARIEB E. N.: Essentials of Human Anatomy and Physiology, 11 ed. Pearson Education, 2015.

MALTi A., BARTOLI A.: Estimating the cook-torrance brdf parameters in-vivo from laparoscopic images. In Proceedings of the Workshop on Augmented Environment in Medical Image Computing and Computer Assisted Intervention (MICCAI) (Nice, France, October 2012).

Malti A., BARtoli A.: Combining conformal deformation and cook-torrance shading for 3-d reconstruction in laparoscopy. IEEE Transactions on Biomedical Engineering 61, 6 (June 2014), 16841692. 
Marescaux J., Clement J. M., Tassetti V., Koehl C., Cotin S., Russier Y., Mutter D., Delingette H., Ayache N.: Virtual reality applied to hepatic surgery simulation: the next revolution. Annals of Surgery 228, 5 (November 1998), 627-34.

MAHY M., EYCKen L., OOSTERLINCK A.: Evaluation of uniform color spaces developed after the adoption of cielab and cieluv. Color Research \& Application 19, 2 (1994), 105-121.

Mescher A. L.: Junqueira's Basic Histology. McGraw-Hill Education, 2017.

Miller G. S., HOFFMAN C. R.: Illumination and reflection maps: simulated objects in simulated and real environments. SIGGRAPH 1984 Advanced Computer Graphics Animation seminar notes (1984).

Marschner S. R., Jensen H. W., Cammarano M., Worley S., HANRAHAN P.: Light scattering from human hair fibers. ACM Trans. Graph. 22, 3 (July 2003), 780-791.

Michael Johnson J., Mahfouz M. R.: Cartilage loss patterns within femorotibial contact regions during deep knee bend. Journal of Biomechanics 49, 9 (sep 2016), 1794-1801.

MlejneK M., Vilanova A., Groller M. E.: Interactive thickness visualization of articular cartilage. In IEEE Visualization 2004 (Oct 2004), pp. 521-527.

Marschner S. R., Westin S. H., LAFOrtune E. P. F., TorRANCE K. E., GREENBERG D. P.: Image-based brdf measurement including human skin. In Proceedings of the 10th Eurographics conference on Rendering (Aire-la-Ville, Switzerland, Switzerland, 1999), EGWR'99, Eurographics Association, pp. 131-144.

Neyret F., CAni M.-P.: Pattern-based texturing revisited. In SIGGRAPH 99 Conference Proceedings (Aug. 1999), ACM SIGGRAPH, Addison Wesley, pp. 235-242.

NGan A., Durand F., MatusiK W.: Experimental analysis of brdf models. In Proceedings of the Sixteenth Eurographics Conference on Rendering Techniques (Aire-la-Ville, Switzerland, Switzerland, 2005), EGSR '05, Eurographics Association, pp. 117-126.

Neyret F., Heiss R., Senegas F.: Realistic rendering of an organ surface in real-time for laparoscopic surgery simulation. The Visual Computer 18, 3 (2002), 135-149.

Nunes A., Maciel A., Cavazzola L., Walter M.: A laparoscopybased method for brdf estimation from in vivo human liver. Medical image analysis 35 (2017), 620-632.

Nicodemus F. E., Richmond J. C., Hsia J. J., Ginsberg I. W., LIMPERIS T.: Radiometry. Jones and Bartlett Publishers, Inc., USA, 1992, ch. Geometrical considerations and nomenclature for reflectance, pp. 94-145.

Olson R., GAYlor J., Everett M.: Skin color, melanin, and erythema. Arch. Dermatol. 108, 4 (1973), 541-544.

Ovalle W., Nahirney P., NetTer F.: Netter's Essential Histology. ClinicalKey 2012. Elsevier Saunders, 2013.

OPPENHEIMER P. E.: Real time design and animation of fractal plants and trees. In ACM SiGGRAPH Computer Graphics (1986), vol. 20, ACM, pp. 55-64.
Omelyanenko N., Slutsky L., Mironov S.: Connective Tissue: Histophysiology, Biochemistry, Molecular Biology. CRC Press, 2016.

PATHAK M.: Functions of melanin and protection by melanin. In Melanin: Its Role in Human Photoprotection (Overland Park, Kansas, USA, 1995), L. Zeise M. C., Fitzpatrick T., (Eds.), Valdenmar Publishing Co., pp. 125-134.

PERLIN K.: An image synthesizer. SIGGRAPH Comput. Graph. 19, 3 (July 1985), 287-296.

PRUSinkiewicz P., Lindenmayer A.: The Algorithmic Beauty of Plants. Springer-Verlag New York, Inc., New York, NY, USA, 1996.

PrAhl S.: Light Transport in Tissue. PhD thesis, The University of Texas at Austin, USA, December 1988.

QIAN K., BAI J., YANG X., PAN J., ZHANG J.: Virtual reality based laparoscopic surgery simulation. In Proceedings of the 21 st ACM Symposium on Virtual Reality Software and Technology (New York, NY, USA, 2015), VRST '15, ACM, pp. 69-78.

Qian K., Bai J., YANG X., PAN J., Zhang J.: Essential techniques for laparoscopic surgery simulation. Computer Animation and Virtual Worlds (2016), n/a-n/a.

Ruhland K., Andrist S., Badler J. B., Peters C. E., Badler N. I., Gleicher M., Mutlu B., MCDonnell R.: Look me in the Eyes: A Survey of Eye and Gaze Animation for Virtual Agents and Artificial Systems. In Eurographics 2014 - State of the Art Reports (2014), Lefebvre S., Spagnuolo M., (Eds.), The Eurographics Association.

Renard P., Alcolea A., Ginsbourger D.: Stochastic versus deterministic approaches. In Environmental Modeling: Finding Simplicity in Complexity (2013), Wainwright J., Mulligan M., (Eds.), John Wiley \& Sons, pp. 133-149.

RAMAMOORTHI R., HANRAHAN P.: An efficient representation for irradiance environment maps. In Proceedings of the 28th Annual Conference on Computer Graphics and Interactive Techniques (New York, NY, USA, 2001), SIGGRAPH '01, ACM, pp. 497-500.

Rовв R. A.: Visualization in biomedical computing. Parallel Computing 25, 13 (1999), 2067 - 2110.

Robb R. A., Ritman E. L., Greenleaf J. F., Sturm R. E., Herman G. T., Chevalier P. A., LiU H. K., Wood E. H.: Quantitative imaging of dynamic structure and function of the heart, lungs and circulation by computerized reconstruction and subtraction techniques. SIGGRAPH Comput. Graph. 10, 2 (July 1976), 246-256.

Spitzer V., ACKerman M. J., Scherzinger A. L., WhitLOCK D.: The visible human male: a technical report. J Am Med Inform Assoc 3, 2 (1996), 118-130.

SATAVA R. M.: Virtual reality, telesurgery, and the new world order of medicine. Journal of Image Guided Surgery 1, 1 (1995), $12-16$.

ShetTy S., BAILEY M.: A physical rendering model for human teeth. In ACM SIGGRAPH 2010 Posters (2010), SIGGRAPH '10, pp. 116:1-116:1. 
SZÉKely G., BrechbüHler C., DuAl J., EnZler R., Hug J., Hutter R., Ironmonger N., KAUer M., Meier V., Niederer P., Rhomberg A., Schmid P., Schweitzer G., Thaler M., Vuskovic V., Tröster G., HAller U., BAJKA M.: Virtual reality-based simulation of endoscopic surgery. Presence: Teleoper. Virtual Environ. 9, 3 (June 2000), 310-333.

Sagar M. A., Bullivant D., Mallinson G. D., Hunter P. J.: A virtual environment and model of the eye for surgical simulation. In Proceedings of the 21st Annual Conference on Computer Graphics and Interactive Techniques (New York, NY, USA, 1994), SIGGRAPH '94, ACM, pp. 205-212.

Stamnes K., Conklin P.: A new multi-layer discrete ordinate approach to radiative transfer in vertically inhomogeneous atmospheres. Journal of Quantum Spectroscopy and Radiative Transfer 31, 3 (1984), 273-282.

S. Chen L., Herman G. T., Reynolds R. A., Udupa J. K.: Surface shading in the cuberille environment. IEEE Computer Graphics and Applications 5, 12 (Dec 1985), 33-43.

Sunguroff A., Greenberg D.: Computer generated images for medical applications. In Proceedings of the 5th annual conference on Computer graphics and interactive techniques (New York, NY, USA, 1978), SIGGRAPH '78, ACM, pp. 196-202.

Szabo G., Gerald A., Pathak M., FitzPatrick T.: Racial differences in the fate of melanosomes in human epidermis. Nature 222, 5198 (06 1969), 1081-1082.

SARni S., MACiel A., Boulic R., Thalmann D.: Evaluation and visualization of stress and strain on soft biological tissues in contact. In Proceedings of the International Conference on Shape Modeling and Applications (SMI 2004) (2004), pp. 255-262.

Spencer R. Van Leeuwen G. V. G. B.: Elucidating the contribution of Rayleigh scattering to the bluish appearance of veins. Journal of Biomedical Optics 23 (2018), 23 - 23 - 17.

STAM J.: An illumination model for a skin layer bounded by rough surfaces. In 12th Eurographics Workshop on Rendering Techniques (2001), Springer-Verlag, pp. 39-52.

SPITZER D., TEN BOSCH J.: The absorption and scattering of light in bovine and human dental enamel. Calcified Tissue International 17, 2 (1975), 129-137.

TEN Bosch J. J., ZIJP J. R.: Optical properties of dentin. In Dentine and Dentine Reactions in the Oral Cavity (1987), IRL Press Ltd, pp. 59-65.

TSAI Y.-T., SHIH Z.-C.: All-frequency precomputed radiance transfer using spherical radial basis functions and clustered tensor approximation. ACM Trans. Graph. 25, 3 (July 2006), 967-976.

T X., SM H., MA C., M Z., AB M., MA M.: Hospital cost implications of increased use of minimally invasive surgery. JAMA Surgery 150, 5 (2015), 489-490.

Tuchin V.: Tissue Optics Light Scattering Methods and Instruments for Medical Diagnosis. The International Society for Optical Engineering, Bellingham, WA, USA, 2000.

UEDA K., FUJISHIRO I.: Adsorptive sph for directable bleeding simulation. In Proceedings of the 14th ACM SIGGRAPH International Conference on Virtual Reality Continuum and Its Applications in Industry (New York, NY, USA, 2015), VRCAI '15, ACM, pp. 9-16.

Ward K., Bertails F., Kim T.-Y., Marschner S. R., CANi M.-P., LIN M. C.: A survey on hair modeling: Styling, simulation, and rendering. IEEE Transactions on Visualization and Computer Graphics 13, 2 (Mar. 2007), 213-234.

WANG L., JACQUeS S., Zheng L.: MCML - Monte Carlo modelling of light transport in multi-layered tissues. Computer Methods and Programs in Biomedicine 47 (1995), 131-146.

W.Y. LAM M., V.G. BARAnoski G.: A Predictive Light Transport Model for the Human Iris. Computer Graphics Forum (2006).

Weyrich T., Matusik W., Pfister H., Bickel B., Donner C., Tu C., McAndless J., Lee J., Ngan A., Jensen H. W., Gross M.: Analysis of human faces using a measurement-based skin reflectance model. ACM Transactions on Graphics 25, 3 (July 2006), 1013-1024.

Worley S.: A cellular texture basis function. In Proceedings of the 23rd Annual Conference on Computer Graphics and Interactive Techniques (New York, NY, USA, 1996), SIGGRAPH '96, ACM, pp. 291-294.

Yim D., Baranoski G., Kimmel B., Chen T., Miranda E.: A cell-based light interaction model for human blood. Computer Graphics Forum 31, 2 (2012), 845-854.

Yan L.-Q., Tseng C.-W., Jensen H. W., Ramamoorthi R.: Physically-accurate fur reflectance: Modeling, measurement and rendering. ACM Trans. Graph. 34, 6 (Oct. 2015), 185:1-185:13.

Yang M., Tuchin V., Yaroslavsky A.: Principles of lightskin interactions. In Light-Based Therapies for Skin of Color (London, 2009), Baron E., (Ed.), Springer-Verlag, pp. 1-44.

Zheng L., Chaudhari A. J., Badawi R. D., Ma K. L.: Using global illumination in volume visualization of rheumatoid arthritis ct data. IEEE Computer Graphics and Applications 34, 6 (Nov 2014), 16-23. 